\title{
ABUNDANCE ANALYSIS OF HE 2148-1247, A STAR WITH EXTREMELY ENHANCED NEUTRON CAPTURE ELEMENTS ${ }^{1}$
}

\author{
Judith G. Cohen, ${ }^{2}$ Norbert Christlieb, ${ }^{3,4}$ Y.-Z Qian, ${ }^{5}$ And G. J. Wasserburg ${ }^{6}$ \\ Received 2002 November 22; accepted 2002 December 30
}

\begin{abstract}
Abundances for 27 elements in the very metal-poor dwarf star HE 2148-1247 are presented, including many of the neutron capture elements. We establish that HE 2148-1247 is a very highly s-process-enhanced star with anomalously high $\mathrm{Eu}$ as well, $\mathrm{Eu} / \mathrm{H} \sim$ half-solar, demonstrating the large addition of heavy nuclei at $[\mathrm{Fe} / \mathrm{H}]=-2.3$ dex. $\mathrm{Ba}$ and $\mathrm{La}$ are enhanced by a somewhat larger factor and reach the solar abundance, while $\mathrm{Pb}$ significantly exceeds it, thus demonstrating the addition of substantial s-process material. $\mathrm{Ba} / \mathrm{Eu}$ is 10 times the solar $r$-process ratio but much less than that of the $s$-process, indicating a substantial $r$-process addition as well. $\mathrm{C}$ and $\mathrm{N}$ are also very highly enhanced. We have found that HE $2148-1247$ is a radial velocity variable; it is probably a small-amplitude long-period binary. The $\mathrm{C}, \mathrm{N}$, and the $s$-process element enhancements were thus presumably produced through mass transfer from a former asymptotic giant branch (AGB) binary companion. The large enhancement of heavy $r$-nuclides also requires an additional source as this is far above any inventory in the interstellar medium at such low $[\mathrm{Fe} / \mathrm{H}]$. We consider that the $s$-process material was added by mass transfer of a more massive companion during its thermally pulsating AGB phase and ending up as a white dwarf. We further hypothesize that accretion onto the white dwarf from the envelope of the star caused accretion-induced collapse of the white dwarf, forming a neutron star, which then produced heavy $r$-nuclides and again contaminated its companion. This mechanism in a binary system can thus enhance the envelope of the lower mass star in $s$ - and $r$-process material sequentially. Through analysis of the neutron capture element abundances taken from the literature for a large sample of very metal-poor stars, we demonstrate, as exemplified by HE 2148-1247, that mass transfer in a suitable binary can be very efficient in enhancing the heavy elements in a star; it appears to be capable of enhancing the $s$-process elements in very metal-poor stars to near the solar abundance but not substantially above it. The yield of $\mathrm{Pb}$ relative to $\mathrm{Ba}$ appears to vary among very metal-poor stars.
\end{abstract}

Subject headings: stars: abundances — stars: chemically peculiar — stars: evolution — stars: Population II On-line material: machine-readable table

\section{INTRODUCTION}

The most metal-deficient stars in the Galaxy provide crucial evidence on the early epochs of star formation, the environments in which various elements were produced, and the production of elements subsequent to the big bang and prior to contributions from lower mass stars to the interstellar medium (ISM). The major existing survey for very metal-poor stars is the HK survey described in detail by Beers, Preston, \& Shectman (1985, 1992). The stellar inventory of this survey has been scrutinized with considerable care over the past decade.

In this paper we report the discovery of a peculiar star with highly enhanced abundances of the heavy neutron capture elements, including Eu. The star HE 2148-1247 was found in the course of exploiting the Hamburg/ESO Survey

\footnotetext{
${ }^{1}$ Based on observations obtained at the W. M. Keck Observatory, which is operated jointly by the California Institute of Technology, the University of California, and the National Aeronautics and Space Administration.

2 Palomar Observatory, Mail Stop 105-24, California Institute of Technology, Pasadena, CA 91125.

${ }^{3}$ Department of Astronomy and Space Physics, University of Uppsala, Box 524, SE-75120 Uppsala, Sweden.

${ }^{4}$ Marie Curie Fellow, on sabbatical leave from Hamburger Sternwarte, Germany.

${ }^{5}$ School of Physics and Astronomy, University of Minnesota, Minneapolis, MN 55455.

${ }^{6}$ Lunatic Asylum, Division of Geological and Planetary Sciences, California Institute of Technology, Pasadena, CA 91125.
}

(HES; Wisotzki et al. 2000; Christlieb et al. 2001) to find very metal-poor stars. We provide a detailed abundance analysis for this star, obtaining results for 27 elements, including many of the neutron capture elements. We give a progress report on our radial velocity monitoring of HE $2148-1247$ and conclude with a discussion of the implications of its extreme anomalies for galactic nucleosynthesis and chemical evolution, with emphasis on the issue of the efficiency of mass transfer in binary systems and a comparison of the observed abundances in HE 2148-1247 to those predicted under various scenarios. We present a new version of accretion-induced collapse in a binary system with heavy $r$-process element production as a plausible explanation for the nucleosynthetic history of HE 2148-1247.

We rely heavily in the present work on the procedures and atomic data for abundance analyses of very metal-poor stars described in our earlier papers reporting the results of the Keck Pilot Program on Extremely Metal-poor Stars from the HES (Cohen et al. 2002; Carretta et al. 2002; Lucatello et al. 2003). At the same time, the spectrum of HE $2148-1247$, as well as those of more than 30 additional very metal-poor stars selected from the HES whose spectra we have recently acquired, was observed with a slight variant of the instrumental configuration utilized for the Keck Pilot Program. Thus, we provide additional details of the procedures to be applied for the analysis of the spectrum of HE $2148-1247$, which will also be used for the spectra of very metal-poor stars now in hand. 
The motivation for studying extremely metal-poor stars using the HES is reviewed in Cohen et al. (2002). When our data set for very metal-poor stars now in hand is analyzed, we will be able to address in detail the issue of the scatter in abundance ratios among various elements from star to star and to constrain the nucleosynthetic processes operating. However, only two of the $\sim 40$ high-dispersion spectra of newly discovered very metal-poor stars from the HES that taken thus far at Keck show easily detected lines of the key element Eu. One of these stars, HE 2148-1247, is such an interesting object that we felt a full analysis should be presented as soon as possible.

\section{OBSERVATIONS}

The star HE 2148-1247, at coordinates R.A.(2000.0) = $21^{\mathrm{h}} 51^{\mathrm{m}} 17^{\mathrm{s}} .8$ and $\delta=-12^{\circ} 33^{\prime} 42^{\prime \prime}$, was selected as a candidate extremely metal-poor star from the HES using automatic spectral classification (Christlieb, Wisotzki, \& Grasshoff 2002). A moderate resolution $(\sim 2 \AA)$ spectrum was taken on 1999 November 18 with the $3.5 \mathrm{~m}$ New Technology Telescope at the European Southern Observatory, La Silla, Chile. ${ }^{7}$ That spectrum confirmed the star to be very metal-poor, and it was therefore observed at the Keck Telescope using the High Resolution Echelle Spectrometer (HIRES; Vogt et al. 1994) on 2001 September 28 and 30. Six $1200 \mathrm{~s}$ exposures were obtained the first night, and five more the second, for a total exposure time of 13,200 s. The star was moved slightly along the slit between each exposure. The observations were carried out with the slit at the parallactic angle.

A spectral resolution of 45,000 was achieved using a 0 ".86 wide slit projecting to 3 pixels in the HIRES focal plane $\mathrm{CCD}$ detector. Because the last order to the red in the spectra taken for the Keck Pilot Program contained essentially no new information for such metal-poor stars, the HIRES configuration was slightly shifted to give up the reddest order, thus allowing the inclusion of one more order to the blue. We thus cover the region from 3805 to $5320 \AA$, with no gaps to $4910 \AA$, and small gaps thereafter to the red as the width of the HIRES CCD is then smaller than the free spectral range of the echelle. We will continue with this new configuration until the HIRES detector is upgraded.

The spectra were exposed such that a minimum signal-tonoise ratio $(\mathrm{S} / \mathrm{N})$ of 100 per spectral resolution element in the continuum at $4500 \AA$ was achieved. This $\mathrm{S} / \mathrm{N}$ calculation utilizes only Poisson statistics, ignoring issues of cosmic ray removal, night-sky subtraction, flattening, etc. (The gain setting of the HIRES CCD detector is $2.4 e^{-} \mathrm{ADU}^{-1}$; to reach the desired $\mathrm{S} / \mathrm{N}$ of 100 per spectral resolution element requires $1390 \mathrm{ADU}$ pixel $^{-1}$.) The sum of the HIRES exposures for HE 2148-1247 has $3000 \mathrm{ADU}_{\text {pixel }}^{-1}$ at the center of the echelle order in the continuum near $4500 \AA$, corresponding to a $\mathrm{S} / \mathrm{N}$ of 145 per spectral resolution element, or 85 per pixel.

This set of HIRES data was reduced using a combination of Figaro scripts and the software package MAKEE. ${ }^{8}$ MAKEE automatically applies heliocentric corrections to each of the individual spectra. The bias removal, flattening,

\footnotetext{
${ }^{7}$ ESO Program ID 64.L-0005(A).

${ }^{8}$ MAKEE was developed by T. A. Barlow specifically for reduction of Keck HIRES data. It is freely available on the World Wide Web at the Keck Observatory home page, http://www2.keck.hawaii.edu.
}

sky subtraction, object extraction, and wavelength solutions with the Th-Ar arc were performed within MAKEE, after which further processing and analysis was carried out within Figaro, where the individual spectra were summed. The continuum fitting to the sum of the individual spectra (already approximately corrected via the mean signal level in the flat-field spectrum) uses a fourth-order polynomial to line-free regions of the spectrum in each order. The degree of the polynomial was reduced in orders with $\mathrm{H}$ and $\mathrm{K}$ of $\mathrm{Ca}$ II or Balmer lines where the fraction of the order available to define the continuum decreased significantly. A scheme of using adjacent orders to help define the polynomial under such conditions is included in the codes. The suite of routines for analyzing Echèlle spectra was written by McCarthy (1988) within the Figaro image processing package (Shortridge 1993).

\subsection{Equivalent Widths}

The search for absorption features present in our HIRES data and the measurement of their equivalent width $\left(W_{\lambda}\right)$ was done automatically with a FORTRAN code, EWDET, developed for a globular cluster project. Details of this code and its features are given in Ramírez et al. (2001). Except in regions affected by $\mathrm{CH}, \mathrm{CN}$, or (rarely) $\mathrm{C}_{2}$ bands, the determination of the continuum level in these very metal-poor stars was easy, and the equivalent widths measured automatically should be quite reliable. Except in regions affected by molecular bands, the crowding of lines is minimal. Hence, we initially use automatic Gaussian fits for $W_{\lambda}$.

The list of lines identified and measured by EWDET is then correlated, taking the radial velocity into account, to the template list of suitable unblended lines with atomic parameters described in $\S 3$ to specifically identify the various atomic lines. Selected wavelength regions (i.e., those strongly affected by molecular bands, where no individual line identification can be regarded as secure or the line as unblended) can be blanked out in this process.

At this point we saw the first hint of the extreme peculiarity of HE 2148-1247, as strong Ba II, La II, Eu II, and Nd II features turned up in the list of automatically identified lines. Selected regions in the spectrum of HE 2148-1247 illustrating some of them are shown in Figure 1. By comparison, HE 2344-2800, analyzed in Cohen et al. (2002) and Carretta et al. (2002), is a "normal" very metal-poor mainsequence turnoff star from the HES with about the same $T_{\text {eff }}, \log (g)$, and $[\mathrm{Fe} / \mathrm{H}]$ as HE 2148-1247; it does not show any of the peculiarities of HE $2148-1247$ to be discussed below. The same spectral regions shown in Figure 1 were extracted from the spectrum of HE 2344-2800 and are displayed in Figure 2 as a comparison.

The automatic identifications were accepted as valid for lines with $W_{\lambda} \geq 15 \mathrm{~m} \AA$. They were checked by hand for all lines with smaller $W_{\lambda}$. The identifications for all the rare earths in the spectrum of HE 2148-1247 were checked by hand. The resulting $W_{\lambda}$ for 159 lines in the spectrum of $\mathrm{HE}$ 2148-1247 are listed in Table 1.

\section{ATOMIC DATA}

We attempt to assemble a complete list of the unblended atomic features in this spectral region that might be seen among our entire set of spectra. We begin with the line list of Carretta et al. (2002), created to support our earlier 

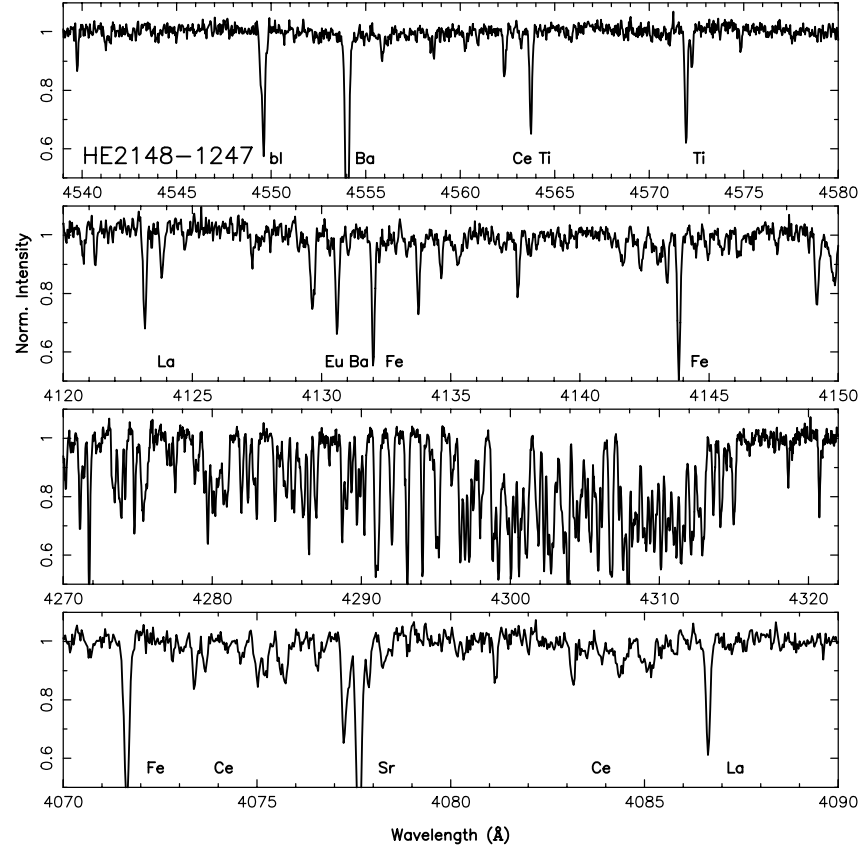

FIG. 1.-Extracts from the spectrum of HE 2148-1247 are shown illustrating the region around the $\mathrm{Ba}$ II line at $4554 \AA$, the Eu II line at $4129 \AA$, the $0-0$ band head of the $\mathrm{G}$ band of $\mathrm{CH}$, and the La II line at $4086 \mathrm{~A}$. The radial velocity of the star has been removed by suitable shifts. Selected strong lines are labeled; the identifications are displaced $0.4 \mathrm{~A}$ to the red of the line center. " $\mathrm{Bl}$ " denotes blends of $\mathrm{Fe}$ and Ti lines.

papers on abundances in extremely metal-poor stars. With the exceptions noted below, we adopt their $g f$-values when available. A few blended lines were deleted from their line list. We then add the unblended lines in the additional bluest
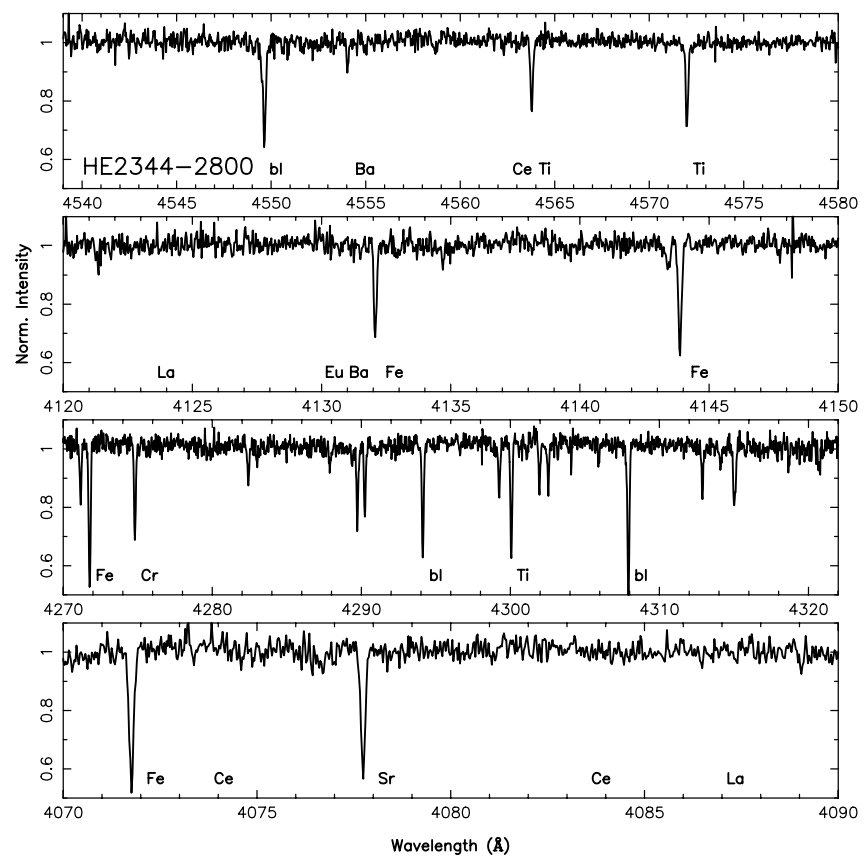

FIG. 2.-Extracts from the spectrum of HE 2344-2800 are shown illustrating the region around the $\mathrm{Ba}$ II line at $4554 \mathrm{~A}$, the Eu II line at $4129 \mathrm{~A}$, the $0-0$ band head of the $\mathrm{G}$ band of $\mathrm{CH}$, and the La II line at $4086 \AA$. The radial velocity of the star has been removed by suitable shifts. The line identifications are those of Fig. 1, except for the region around the $\mathrm{CH} \mathrm{G}$ band head, and are shown displaced $0.4 \AA$ to the red of the line center.
TABLE 1

$W_{\lambda}$ FOR LiNes IN THE SPectrum OF HE 2148-1247

\begin{tabular}{|c|c|c|c|c|c|}
\hline Species & $\begin{array}{l}\text { Wavelength } \\
\text { (̊) }\end{array}$ & $\begin{array}{l}\mathrm{EP} \\
(\mathrm{eV})\end{array}$ & $\begin{array}{c}\log (g f) \\
100\end{array}$ & $\begin{array}{c}C_{6}{ }^{\mathrm{a}} \\
\left(\mathrm{Hz} \mathrm{cm}^{6}\right)\end{array}$ & $\begin{array}{c}W_{\lambda} \\
(\mathrm{m} \AA)\end{array}$ \\
\hline $\operatorname{Mg}_{\mathrm{I}} \ldots \ldots . .$. & 4057.52 & 4.34 & -1.200 & $0.630 \mathrm{E}-29$ & 14.0 \\
\hline $\operatorname{Mg}_{1} \ldots \ldots . .$. & 4167.28 & 4.34 & -1.000 & $0.630 \mathrm{E}-29$ & 39.5 \\
\hline $\operatorname{Mg}_{\mathrm{I}} \ldots \ldots .$. & 4703.00 & 4.34 & -0.670 & $0.132 E-29$ & 46.3 \\
\hline $\operatorname{Mg}_{\mathrm{I}} \ldots \ldots . .$. & 5172.70 & 2.71 & -0.380 & $0.138 \mathrm{E}-30$ & 130.7 \\
\hline $\mathrm{Mg}_{\mathrm{I}} \ldots \ldots . .$. & 5183.62 & 2.72 & -0.160 & $0.138 \mathrm{E}-30$ & 159.6 \\
\hline $\mathrm{Al}$ I ............. & 3961.52 & 0.00 & -0.340 & $0.631 \mathrm{E}-30$ & 78.4 \\
\hline Si I .......... & 3905.53 & 1.91 & -1.000 & $0.190 \mathrm{E}-30$ & 149.5 \\
\hline Ca I ......... & 4226.74 & 0.00 & 0.240 & $0.589 E-31$ & 137.2 \\
\hline $\mathrm{Ca}$ I ......... & 4318.66 & 1.90 & -0.210 & $0.589 \mathrm{E}-30$ & 29.7 \\
\hline Ca I ......... & 4425.44 & 1.88 & -0.360 & $0.589 E-30$ & 26.6 \\
\hline
\end{tabular}

Note.-Table 1 is published in its entirety in the electronic edition of the Astrophysical Journal. A portion is shown here for guidance regarding its form and content

a This is either the value of $C_{6}$ for van der Waals broadening or the multiplier to be applied to the Unsöld 1955 approximation for $C_{6}$.

HIRES order. The transition probabilities for these were taken from the NIST Atomic Spectra Database Version 2.0 (NIST Standard Reference Database 78; see Wiese, Smith, \& Miles 1969; Wiese, Fuhr, \& Deters 1996; Martin, Fuhr, \& Wiese 1988; Fuhr, Martin, \& Wiese 1988). In the Appendix to Carretta et al. (2002) we give a comparison of our adopted transition probabilities for our initial work with those of NIST. Very good agreement was noted for all ions except $\mathrm{Mg} \mathrm{I}$, $\mathrm{Ti}$ II, and $\mathrm{Fe}$ II, where larger dispersions occurred. The additional lines resulting from the extra blue HIRES order included here do not contain any lines from these three ions. We adopted the $g f$-values of Lawler \& Dakin (1989) for Sc II (which agree with those of NIST), as well as those of Biémont et al. (2000) for Pb I.

Since we already have more than 30 spectra of very metalpoor stars covering a range of metallicity with $[\mathrm{Fe} / \mathrm{H}] \leq-2$ $\mathrm{dex}^{9}$ taken with the same instrumental configuration, we also searched for the strongest clean lines in this spectral region of several ions missing from our earlier papers, which we might hope to detect. Lines of $\mathrm{Ni}$ I and $\mathrm{Co}$ I were picked up in this way in the spectrum of HE 2148-1247 and added, again with $g f$-values from NIST. We then added into the template line list the strongest expected lines of the rare earths using the line list and $g f$-values from Sneden et al. (1996). This produced a template list of 259 lines, 182 of which arise from species through atomic number $28(\mathrm{Ni})$, with 15 lines from $\mathrm{Sr}, \mathrm{Y}$ or $\mathrm{Zr}$, three from $\mathrm{Ba}$, and the remainder from the singly ionized rare earths with the strongest expected lines ( $\mathrm{La}, \mathrm{Ce}, \mathrm{Pr}, \mathrm{Nd}, \mathrm{Sm}, \mathrm{Eu}, \mathrm{Gd}$, and $\mathrm{Dy}$ ).

\subsection{Damping Constants}

Values of the collisional broadening parameter $C_{6}$ for van der Waals broadening are available from detailed fits to solar line profiles for the $\mathrm{Al}$ I doublet at $3950 \AA$ from Baumüller \& Gehren (1996), for three of the five Mg I lines of interest from Zhao, Butler, \& Gehren (1998), and for the $4554 \AA \mathrm{Ba}$ II line (one of the three lines of interest of this ion) from Gehren et al. (2001; chosen in preference to

\footnotetext{
${ }^{9}$ The standard nomenclature is adopted; the abundance of element $\mathrm{X}$ is given by $\epsilon(\mathrm{X})=N(\mathrm{X}) / N(\mathrm{H})$ on a scale where $N(\mathrm{H})=10^{12} \mathrm{H}$ atoms. Then $[\mathrm{X} / \mathrm{H}]=\log _{10}[N(\mathrm{X}) / N(\mathrm{H})]-\log _{10}[N(\mathrm{X}) / N(\mathrm{H})]_{\odot}$, and similarly for $[\mathrm{X} / \mathrm{Fe}]$.
} 
the even larger value of $C_{6}$ given for this line in Mashonkina, Gehren, \& Bikmaev 1999). When such are available, these values are adopted.

Damping constants for the remaining lines of the species $\mathrm{Mg}$ I, $\mathrm{Al}$ I, $\mathrm{Si}$ I, $\mathrm{Ca}$ I, $\mathrm{Sr}$ II, and $\mathrm{Ba}$ II that might be strong enough for collisional broadening to be important are taken from the recent compilation of collisional damping parameters calculated from theory by Barklem, Piskunov, \& O'Mara (2000). The damping constants for all Fe I and Fe II lines were set to twice that of the Unsöld approximation for van der Waals broadening following Holweger et al. (1991). The damping constant $C_{6}$ is set to twice that of the Unsöld approximation for van der Waals broadening for all other lines, none of which are strong enough to be affected by the choice of $C_{6}$. Note that the above prescription is applied to all spectral lines irrespective of the value of their measured equivalent width.

\subsection{Hyperfine Structure}

Many of the ions considered here have lines that show appreciable hyperfine structure (hfs). In some cases, the additional complication of multiple stable isotopes is also present. We adopt the hfs patterns of McWilliam (1998) for $\mathrm{Ba}$ II. These are calculated with the solar $r$-process isotope distribution. We also calculated the Ba II hfs patterns from the $r$-process patterns using the isotopic distributions given by Arlandini et al. (1999). We use the hfs and atomic constants of Lawler, Bonvallet, \& Sneden (2001a) for La II and of Lawler et al. (2001b) for Eu II. We assumed equal abundances for the two stable isotopes of $\mathrm{Eu},{ }^{151} \mathrm{Eu}$ and ${ }^{153} \mathrm{Eu}$, which is (approximately) the solar value and that characteristic of the $r$-process, as discussed in Käppeler, Beer, \& Wisshak (1989). We calculate the hfs patterns for the relevant Co I lines from data in Pickering (1996) and Pickering \& Thorne (1996). The hfs patterns for the relevant Mn lines were calculated from the data in Booth, Shallis, \& Wells (1983). There are four lines of Sc II on the template list. For one of these, the hfs pattern was available from Prochaska et al. (2000). For the other three, we calculated the hfs patterns using constants from Arnesen et al. (1982), Mansour et al. (1989), and Villemoes et al. (1992). For the single V I line in the template list (the only clean strong line of $\mathrm{V}$ in the blue spectral region), which is not detected in $\mathrm{HE}$ 2148-1247, we could only locate hfs parameters for the lower level (Childs et al. 1979). We adopted parameters for the upper level comparable to the smallest splitting found by Childs et al. (1979) for any of the levels of V I they studied to calculate the hfs pattern for this line.

\section{SOLAR ABUNDANCES}

A number of major advances in abundance analyses have occurred over the past decade, reflecting progress in atomic physics and computing power. It is now possible to calculate non-LTE corrections more accurately using more realistic model atoms. Furthermore, realistic three-dimensional radiative-hydrodynamical convection simulations have begun to become available (Asplund et al. 2000) and to be applied to abundance determinations in a small number of key situations. Allende-Prieto, Lambert, \& Asplund (2001, 2002) discuss the case of $\mathrm{C}$ and of $\mathrm{O}$ in the Sun.

We assume the solar abundances of Anders \& Grevesse (1989), as updated in Grevesse \& Sauval (1998). Those of
Anders \& Grevesse (1989) have been derived using classical one-dimensional abundance analyses of carefully selected photospheric lines, with many elements determined from meteoritic values relative to Si. Holweger (2001) provides updates for the solar abundances of several key elements including not only small non-LTE corrections (his maximum $|\Delta(\mathrm{NLTE})|$ is $0.045 \mathrm{dex})$ but also "granulation" corrections resulting from the inadequacies of a classical one-dimensional model given the reality of convection. His maximum $|\Delta(\operatorname{gran})|$ is $0.070 \mathrm{dex}$, for nitrogen, where the very high excitation of the $\mathrm{N}_{\mathrm{I}}$ lines used in the solar analysis produces high sensitivity to fluctuations in temperature.

Non-LTE corrections appropriate for low-metallicity stars are provided in Table 3 when available, but we retain the one-dimensional model atmosphere limitation. Thus, for example, the solar $\mathrm{N}$ abundance adopted here includes the -0.07 dex granulation correction, but we do not apply it to the $\mathrm{N}$ abundance in HE 2148-1247, obtained from a CN band.

We assume that the absolute scale of the adopted set of $g f$-values is correct over the range of line strength from the lines we use here for very metal-poor stars with high $g f$ values to the much weaker lines normally used to determine solar abundances. Ramírez \& Cohen (2003) have checked this for a set of lines used to analyze stars in the Galactic globular cluster M5, which at $[\mathrm{Fe} / \mathrm{H}] \sim-1.3$ dex is significantly more metal-rich than the stars considered here. They successfully reproduced the standard solar abundance for the atomic species in common with the present work to within \pm 0.10 dex with the exception of $\mathrm{Ca}$ I, which had a discrepancy of 0.16 dex.

As we use the $g f$-values and the hyperfine structure constants of Lawler et al. (2001a) for La II, we use their solar La abundance as well, which is slightly different from that given by either of the major compilations cited above. The Lawler et al. (2001b) deduced solar Eu abundance is in good agreement with that of Anders \& Grevesse (1989). The same situation holds for $\mathrm{Pb}$ I, where the new $g$-values of Biémont et al. (2000) produce a small shift in the Grevesse \& Sauval (1998) solar abundance. The adopted solar abundances are included in column (6) of Table 3.

\section{RADIAL VELOCITY MONITORING}

After the first spectra revealed the peculiar nature of HE $2148-1247$, we began a radial velocity monitoring program, attempting to observe the star on each of our subsequent Keck/HIRES runs.

The wavelength scale of our spectra are set by observations of a Th-Ar arc at least twice per night, together with appropriate heliocentric corrections. The radial velocity measurement scheme developed for very metal-poor stars in the HES relies upon a set of accurate laboratory wavelengths for very strong isolated features within the wavelength range of the HIRES spectra. The wavelengths were taken from the NIST Atomic Spectra Database Version 2.0 (NIST Standard Reference Database 78). Using an approximate initial $v_{r}$, the list of automatically detected lines, restricted to the strongest detected lines only, generated in the course of measuring the equivalent widths of the lines (see $\S 2.1$ ) in the spectrum of HE 2148-1247 was then searched for each of these features. A $v_{r}$ for each line was determined from the central wavelength of the best-fit Gaussian, and the average of these, with a $2.5 \sigma$ clipping 
reject cycle, defined the $v_{r}$ for the star. Very metal-poor stars that have been observed repeatedly by George Preston (see Preston \& Sneden 2001) are used as radial velocity standards. The observations of these stars are treated identically as the sample stars; they serve to monitor the accuracy of the analysis.

Since we are interested in small velocity changes, we have taken several steps to increase the accuracy of these measurements. The two laboratory lines between 4270 and 4330 $\AA$ are omitted if the star shows strong $\mathrm{CH}$. We have also used the spectra of five bright metal-poor stars without strong $\mathrm{CH}$ to determine the small mean offsets between the $v_{r}$ measured from each of the laboratory lines. We set the absolute scale defining the lab wavelengths of the two unblended lines of the $\mathrm{Mg}$ triplet as 5172.684 and 5183.604 $\AA$. With that definition, the offsets range from +4.3 to -2.7 $\mathrm{km} \mathrm{s}^{-1}$, with only six of them exceeding $+1 \mathrm{~km} \mathrm{~s}^{-1}$ or less than $-1 \mathrm{~km} \mathrm{~s}^{-1}$. Such differences might arise from small errors in the laboratory wavelength or repeatable local errors in the arc-fitting procedures. With these modifications, we are able to achieve a $1 \sigma \mathrm{rms}$ error for the mean $v_{r}$ of a bright star of $0.15 \mathrm{~km} \mathrm{~s}^{-1}$, and of $\leq 0.3 \mathrm{~km} \mathrm{~s}^{-1}$ for all but the faintest of the HES stars for spectra of the quality described in $\S 2$.

Table 2 lists the results to date for the two stars from the HES that we are currently monitoring, as well as for HD 186478, a C-enhanced metal-poor giant star (McWilliam et al. 1995). (This is one of the stars suggested by G. Preston as a radial velocity standard.) In addition to HE 2148-1247, we are monitoring HE 0007-1832, because when first observed with HIRES in 2001 September, it showed slightly resolved spectral lines. This star has weaker absorption features than does HE 2148-1247. The errors in the table are the $1 \sigma$ rms error in the mean velocity, computed assuming Gaussian statistics from the observed dispersion about the mean and the number of lab lines matched. With HIRES at Keck, 1 pixel is $2.0 \mathrm{~km} \mathrm{~s}^{-1}$, and a spectral resolution element is $\sim 3$ pixels, so a $4 \mathrm{~km} \mathrm{~s}^{-1}$ change in $v_{r}$ is equivalent to a 2 pixel shift in the line center location for each line.

From the data available thus far (three HIRES runs at roughly 6 month intervals over a year), we find that $\mathrm{HE}$ 0007-1832 shows no sign of radial velocity changes. There is some indication that HD 186478 may have a variable

TABLE 2

Radial Velocity MONitoring

\begin{tabular}{|c|c|c|c|c|}
\hline Star & $\mathrm{HJD}^{\mathrm{a}}$ & $\begin{array}{c}v_{r}(\text { Helio }) \\
\left(\mathrm{km} \mathrm{s}^{-1}\right)\end{array}$ & $\begin{array}{c}\sigma \\
\left(\mathrm{km} \mathrm{s}^{-1}\right)\end{array}$ & $\begin{array}{l}\text { Exposure } \\
\text { Time } \\
\text { (s) }\end{array}$ \\
\hline HE $0007-1832 \ldots \ldots .$. & 181.93 & +27.3 & 0.8 & 4800 \\
\hline HE $0007-1832 \ldots \ldots$. & 182.87 & +28.2 & 0.8 & 3600 \\
\hline HE $0007-1832 \ldots \ldots$. & 545.93 & +26.4 & 0.9 & 2400 \\
\hline HE $2148-1247 \ldots \ldots$. & 180.72 & +41.3 & 0.5 & 7200 \\
\hline HE $2148-1247 \ldots \ldots$. & 182.77 & +41.6 & 0.4 & 6000 \\
\hline HE $2148-1247 \ldots \ldots$. & 397.12 & +38.3 & 0.4 & 1200 \\
\hline HE $2148-1247 \ldots \ldots$. & 544.78 & +36.8 & 0.5 & 3600 \\
\hline HD $186478 \ldots \ldots \ldots \ldots$ & 179.71 & +25.7 & 0.6 & 20 \\
\hline HD $186478 \ldots \ldots \ldots \ldots$ & 544.77 & +31.2 & 0.5 & 50 \\
\hline HD $186478 \ldots \ldots \ldots \ldots$ & 545.76 & +31.0 & 0.5 & 50 \\
\hline HD $186478 \ldots \ldots \ldots \ldots$ & 546.76 & +31.3 & 0.6 & 50 \\
\hline
\end{tabular}

a Heliocentric Julian date $-2,452,000.00$. radial velocity, ${ }^{10}$ while the radial velocity of HE $2148-1247$ is definitely variable and is probably a small-amplitude long-period binary. Radial velocity monitoring will continue for these three stars.

\section{STELLAR PARAMETERS}

The procedure used to derive $T_{\text {eff }}$ estimates for $\mathrm{HE}$ $2148-1247$ is fully explained in $\S \S 4-6$ of Cohen et al. (2002). Very briefly, $T_{\text {eff }}$ is derived from broadband colors, taking the mean estimates deduced from the dereddened $V-K$ and $V-J$ colors, where the infrared colors are from 2MASS (Skrutskie et al. 1997). Measurements on both the $1.5 \mathrm{~m}$ telescope at Palomar Mountain and on the Swope Telescope at the Las Campanas Observatory yield $B=$ $15.233 \pm 0.015, V=14.780 \pm 0.023 \mathrm{mag}$ for $\mathrm{HE} 2148-$ 1247. We used the grid of predicted broadband colors and bolometric corrections of Houdashelt, Bell, \& Sweigart (2000), based on the MARCS stellar atmosphere code (Gustafsson et al. 1975) and corrected the colors for reddening by adoption of the extinction maps of Schlegel, Finkbeiner, \& Davis (1998) to find $T_{\text {eff }}=6380 \mathrm{~K}$. This constrains HE 2148-1247 to either be close to the mainsequence turnoff or on the horizontal branch. The latter is ruled out by the broad, pressure-broadened Balmer lines visible in the Keck spectra. We therefore adopt the mainsequence turnoff $\log (g)$ of 3.9 dex, which was derived from the $12 \mathrm{Gyr},[\mathrm{Fe} / \mathrm{H}]=-2.3$ isochrone of $\mathrm{Yi}$ et al. (2001). There is little sensitivity to the choice of $[\mathrm{Fe} / \mathrm{H}]$ at this $T_{\text {eff }}$; if the isochrone for $[\mathrm{Fe} / \mathrm{H}]=-3.3$ is used instead, then the derived $\log (g)$ becomes 4.0 dex. The resulting stellar parameters are $T_{\text {eff }}=6380 \mathrm{~K}, \log (g)=3.9$ dex and we assumed $[\mathrm{Fe} / \mathrm{H}]=-2.5$ dex in this step of our analysis.

\section{ABUNDANCE ANALYSIS-ISOLATED LINES}

We carried out a classical LTE analysis using the equivalent widths measured for individual absorption lines. We employ the grid of stellar atmospheres from Kurucz (1993; with no overshooting) and a current version of the MOOG code (Sneden 1973). Holding $T_{\text {eff }}$ and $\log (g)$ fixed, the final overall metallicities $[\mathrm{M} / \mathrm{H}]$ for $\mathrm{HE} 2148-1247$ were obtained iteratively, by matching observed $W_{\lambda}$ with the synthetic ones computed by integrating the equation of radiative transfer at different wavelengths along each line for the flux. The microturbulent velocity, $v_{t}$, of $1.7 \pm 0.2 \mathrm{~km} \mathrm{~s}^{-1}$, was derived by eliminating any trend in derived abundances of Fe I lines with the expected $W_{\lambda}$ (see Magain 1984). The final abundances are given in Table 3. They were derived by interpolating to the desired $T_{\text {eff }}$ and $\log (g)$ of $\mathrm{HE}$ 2148-1247 from the four nearest model atmospheres in the grid with $[\mathrm{Fe} / \mathrm{H}]=-2.5 \mathrm{dex}$.

As described above in $\S 2.1$, our line list was created automatically (for lines with $W_{\lambda}>15 \mathrm{m \AA}$ ) from the template list of unblended lines. Subsequently, we examined the residuals from the mean abundance for lines of each element. Those lines that gave very discrepant results were checked by hand and were eliminated if they appeared blended or there was some other problem. A relatively small number of lines were eliminated in this way.

\footnotetext{
${ }^{10} \mathrm{G}$. Preston advises that his six observations of HD 186478 covered a time span of only 90 days and showed a $1 \sigma$ rms about the mean of $0.69 \mathrm{~km} \mathrm{~s}^{-1}$.
} 
TABLE 3

ABUNDANCES IN HE 2148-1247

\begin{tabular}{|c|c|c|c|c|c|}
\hline $\begin{array}{l}\text { Species } \\
\text { (1) }\end{array}$ & $\begin{array}{l}\text { Number of Lines } \\
\text { (2) }\end{array}$ & $\begin{array}{c}\log [\epsilon(X)]^{a} \\
(\operatorname{dex}) \\
(3)\end{array}$ & $\begin{array}{c}\Delta{\text { (non-LTE })^{\mathrm{b}}}^{(\mathrm{dex})} \\
(4)\end{array}$ & $\begin{array}{c}\langle[\mathrm{X} / \mathrm{Fe}]\rangle \\
(\mathrm{dex}) \\
(5)\end{array}$ & $\begin{array}{c}\text { Solar } \log [\epsilon(\mathrm{X})]^{\mathrm{c}} \\
(\mathrm{dex}) \\
(6)\end{array}$ \\
\hline $\mathrm{C}(\mathrm{CH})^{\mathrm{d}} \ldots \ldots$ & Syn & $8.08(0.2)$ & & 1.91 & 8.56 \\
\hline $\mathrm{N}(\mathrm{CN}) \ldots \ldots \ldots$ & Syn & $7.25(0.3)$ & & 1.65 & 7.92 \\
\hline $\operatorname{Mg}_{\mathbf{I}} \ldots \ldots \ldots \ldots$ & 5 & $5.72(0.25)$ & +0.15 & 0.50 & 7.54 \\
\hline Al I .............. & 1 & $3.39(\ldots)$ & $+0.6^{\mathrm{e}}$ & -0.76 & 6.47 \\
\hline Si I................. & 1 & $5.92(\ldots)$ & & 0.69 & 7.55 \\
\hline Ca I ............. & 5 & $4.49(0.21)$ & & 0.45 & 6.36 \\
\hline Sc II ............. & 3 & $1.42(0.24)$ & & 0.59 & 4.99 \\
\hline Ti I ................. & 3 & $3.22(0.17)$ & & 0.55 & 4.99 \\
\hline Ti II ............. & 13 & $3.20(0.13)$ & & 0.53 & 4.99 \\
\hline Cri.............. & 3 & $3.01(0.02)$ & & -0.35 & 5.67 \\
\hline Mn I ............ & 2 & $2.46(0.06)$ & & -0.61 & 5.39 \\
\hline Fe I.............. & 58 & $5.13(0.20)$ & +0.10 & 0.00 & 7.45 \\
\hline Fe II............ & 10 & $5.17(0.14)$ & 0.00 & 0.04 & 7.45 \\
\hline Co I................ & 3 & $2.81(0.20)$ & & 0.21 & 4.92 \\
\hline Ni I............. & 2 & $3.99(0.10)$ & & 0.06 & 6.25 \\
\hline Sr II ............. & 2 & $1.34(0.02)$ & & 0.76 & 2.90 \\
\hline Y II .............. & 5 & $0.75(0.12)$ & & 0.83 & 2.24 \\
\hline $\mathrm{Zr}$ II............. & 3 & $1.75(0.18)$ & & 1.47 & 2.60 \\
\hline Ba II ............. & 3 & $2.17(0.07)$ & +0.15 & 2.36 & 2.13 \\
\hline La II ............ & 6 & $1.10(0.20)$ & & 2.38 & 1.14 \\
\hline Ce II ............ & 7 & $1.51(0.20)$ & & 2.28 & 1.55 \\
\hline Pr II .............. & 3 & $0.91(0.30)$ & & 2.52 & 0.71 \\
\hline Nd II............. & 9 & $1.45(0.22)$ & & 2.27 & 1.50 \\
\hline Sm II............ & 2 & $0.67(0.11)$ & & 1.99 & 1.00 \\
\hline Eu II ............ & 3 & $0.17(0.10)$ & -0.10 & 1.98 & 0.51 \\
\hline Gd II............ & 4 & $1.04(0.10)$ & & 2.23 & 1.12 \\
\hline Dy II............. & 3 & $0.95(0.30)$ & & 2.16 & 1.10 \\
\hline $\mathrm{Pb}$ II ............. & syn & $2.8(0.2)$ & & 3.12 & 2.00 \\
\hline Th II ............ & syn & $-0.5(+0.2,-\infty)^{f}$ & & 1.73 & 0.09 \\
\hline
\end{tabular}

a The $1 \sigma$ errors for $\log (\epsilon)$ are given in parentheses.

b The non-LTE corrections are derived from the calculations referenced in $\S 7.1$.

c The solar abundances we adopt are described in $\S 4$.

d Synthesis of the $\mathrm{CH}$ band shows ${ }^{12} \mathrm{C} /{ }^{13} \mathrm{C} \sim 10$; see text.

e The non-LTE correction for the resonance doublet of $\mathrm{Al} I$ is used throughout this paper.

$\mathrm{f}$ The detection of Th is not secure. See the text for details.

\subsection{Ionization Equilibria and Non-LTE}

We detected two states of ionization for both $\mathrm{Fe}$ and $\mathrm{Ti}$ and compared the abundances of the neutral and singly ionized species in each case. Since there are, in principle, a large number of detected $\mathrm{Fe} \mathrm{I}$ lines, we ignore any $\mathrm{Fe} \mathrm{I}$ line with $W_{\lambda}>150 \mathrm{~mA}$. However, there are in fact no Fe I lines in the spectrum of HE 2148-1247 in this wavelength range that are that strong (see Table 1). The comparison is excellent in both cases, with $\log [\epsilon(\mathrm{Fe}$ II $)]-\log \left[\epsilon\left(\mathrm{Fe}_{\mathrm{I}}\right)\right]=-0.04$ dex (with $10 \mathrm{Fe}$ II lines detected). For $\mathrm{Ti}$, where there are only three weak Ti I lines detected, this difference is -0.02 dex. This is remarkably good agreement. Furthermore, the excitation temperature, which we define here as $T_{\text {eff }}$ of the model atmosphere for which the abundance deduced from the set of $58 \mathrm{Fe} \mathrm{I}$ lines is independent of $\chi$, the excitation potential of the lower level, is found to be $6480 \mathrm{~K}$, a value that is in very good agreement with $T_{\text {eff }}$ as determined from broadband colors.

This suggests, as does the $\mathrm{Fe}$ ionization equilibrium found for the sample of extremely metal-poor stars studied by Carretta et al. (2002), that non-LTE does not significantly alter the results of a classical abundance analysis such as presented here. The theoretical situation is somewhat unclear, as the results of recent theoretical analyses (Gratton et al. 1999; Thévenin \& Idiart 1999) disagree on the amplitude to be expected. Gratton et al. (1999) found that NLTE corrections for Fe lines are very small in dwarfs of any $T_{\text {eff }}$, and only small corrections $(<0.1$ dex $)$ are expected for stars on the red giant branch. Thévenin \& Idiart (1999) found that NLTE corrections become more important as $[\mathrm{Fe} / \mathrm{H}]$ decreases, being about 0.2 dex for stars with $[\mathrm{Fe} / \mathrm{H}] \sim-1.25 \mathrm{dex}$, and that ionized lines are not significantly affected by NLTE. Very recently, Gehren et al. (2001) and Gehren, Korn, \& Shi (2001) have carefully calculated the kinetic equilibrium of $\mathrm{Fe}$ and present in Korn \& Gehren (2003) a critique of earlier calculations. They suggest that non-LTE corrections intermediate between the above sets of values are appropriate for Fe I.

Mashonkina et al. (1999) and Gehren et al. (2001) calculate non-LTE for Ba II and Eu II in low-metallicity stars; corrections appropriate for HE 2148-1247 for these elements from their work are in the range -0.1 to $+0.1 \mathrm{dex}$, while Zhao \& Gehren (2000) find small non-LTE corrections for Mg I in very metal-poor stars. The $3970 \AA$ doublet of $\mathrm{Al}$ I, the only lines of this species detected in our spectra, is an exception. The calculated non-LTE corrections of Baumüller \& Gehren (1997) are large, about +0.6 dex for 
HE 2148-1247, while only +0.05 dex is predicted for the Sun. The validity of these large non-LTE corrections for this Al I doublet has been verified by studies of bright field stars using the resonance lines as

compared to less affected subordinate lines.

On this basis, we choose to include non-LTE corrections only for $\mathrm{Al} \mathrm{I}$ in the remainder of this paper. However, column (4) of Table 3 gives the non-LTE corrections appropriate to HE 2148-1247 for each species, when available, from the sources cited above.

\subsection{Ba and the Rare Earths}

The prominence of lines of $\mathrm{Ba}$ and of the rare earths in the spectrum of HE 2148-1247 is illustrated in Figure 1. The Ba II, La II, and Nd II lines are stronger than those of the Sun, even though this star has a very low $[\mathrm{Fe} / \mathrm{H}]$ and is somewhat hotter than the Sun, while the Eu II lines detected are slightly weaker than the corresponding solar lines. The $\mathrm{Ba}$ II lines are the strongest lines in this spectral region, excluding only $\mathrm{H}$ and $\mathrm{K}$ of $\mathrm{Ca}$ II and the Balmer lines. $\mathrm{Ba}$ is a difficult element to analyze, because its lines are so strong that the choice of damping constants and hfs become important. However, we believe, from the comparisons of independent analyses of slightly more metal-rich and slightly cooler globular cluster giants described in Ramírez \& Cohen (2003), that our analysis should be correct to within 0.2 dex. Use of pure $s$-process isotopic distributions in calculating the hfs for the $\mathrm{Ba}$ II lines would decrease the deduced $\mathrm{Ba}$ abundance in HE $2148-1247$ by 0.08 dex.

Among the rare earths detected, the abundance of $\mathrm{Pr}$ is the most uncertain, as all the lines are very weak (see Table 1). For Sm, the $4318 \AA$ line is a very solid detection and is seen in the spectra of HE 2148-1247 taken in 2002 September for radial velocity monitoring as well. The same holds for the $4103 \AA$ line of Dy II. All of the Gd II lines seen are very weak but are definitely real; they too are visible in the 2002 September spectra of this star.

\subsection{Sensitivity Study}

The $1 \sigma$ rms dispersions for the values of $\log [\epsilon(\mathrm{X})]$ given in Table 4 contain contributions from various sources of uncertainties in the stellar parameters as well as in the atomic data. The errors in $[\mathrm{X} / \mathrm{Fe}]$ are typically somewhat smaller than for $\log (\epsilon)$ because the contribution from the uncertainties in the stellar parameters is partially canceled in forming the abundance ratios to Fe; if multiple lines of an ion have been detected, that can also lead to significantly smaller errors.

Note that the sensitivity to changes in $v_{t}$ for those species where hyperfine corrections have been utilized are small. However, if no hfs corrections are used, the sensitivity to changes in $v_{t}$ is increased substantially, up to 0.10 dex for a change of $0.2 \mathrm{~km} \mathrm{~s}^{-1}$, twice the value typical of elements with only strong lines such as Si I.

TABLE 4

Sensitivity of AbUndances

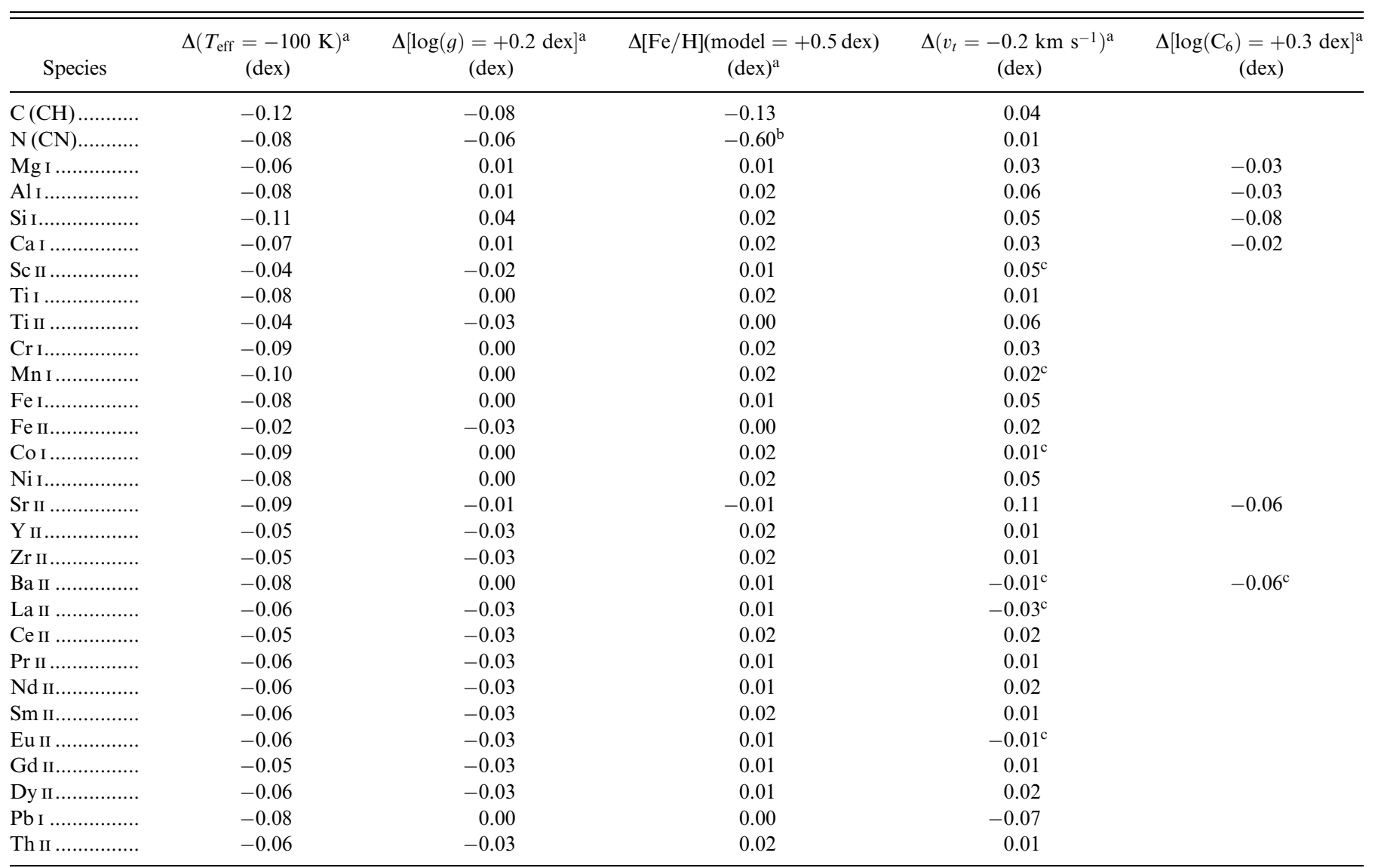

a These are the changes in $\log [\epsilon(\mathrm{X})]$, not in $[\mathrm{X} / \mathrm{Fe}]$. To calculate the latter, subtract $\Delta(\mathrm{Fe})$ from $\Delta(\mathrm{X})$ for a specific parameter, i.e., $T_{\text {eff }}, \log (g)$, etc.

$\mathrm{b}$ This value for $\mathrm{CN}$ is calculated for constant $\mathrm{C} / \mathrm{Fe}$.

${ }^{\mathrm{c}}$ Hyperfine structure corrections have been applied in each case. 


\section{ABUNDANCES FROM SPECTRAL SYNTHESES}

The abundances of $\mathrm{C}, \mathrm{N}, \mathrm{Th}$, and $\mathrm{Pb}$, as well as the ratio of ${ }^{12} \mathrm{C} /{ }^{13} \mathrm{C}$, were derived from spectral syntheses. We generated a line list for each region synthesized by adding the strongest atomic lines in the wavelength interval involved to the relevant molecular line list. We use atomic $g f$-values from NIST, when available, or from Kurucz's (1995) ${ }^{11}$ atomic line database. As a first step in each case, the solar spectrum was synthesized and compared to that observed by Wallace, Hinkle, \& Livingston $(1998)^{12}$ with the FTS at the National Solar Observatory; this solar spectrum is available on-line in digital form. This was done to verify that the final line list for each spectral region can reproduce the solar spectrum with the nominal solar abundances. Because of the extreme enhancement of the neutron capture elements in HE 2148-1247, the successful completion of a synthesis that matches the solar spectrum is no guarantee of a line list sufficiently complete to reproduce the spectrum of $\mathrm{HE}$ 2148-1247. In many cases, additional rare earth lines had to be added to the line list even after the solar spectrum had been reasonably well matched. In all the syntheses, the abundances of the various elements with lines within that spectral region, with the exception of the element being tested, were set to those in Table 3.

\subsection{Carbon}

The $\mathrm{C}$ abundance was determined from syntheses of the $G$ band of $\mathrm{CH}$. The molecular line data for $\mathrm{CH}$, including the $g f$-values and the isotope shifts, were taken from Jorgensen (1994) and Jorgensen et al. (1996). The synthesis was carried out first with an initial guess at ${ }^{12} \mathrm{C} /{ }^{13} \mathrm{C}$, then with the value determined from the spectra of HE 2148-1247. However, the main band head of the $G$ band at $4305 \AA$ was not used because of concerns about continuum placement given the strength of the band and the relatively short length of the echelle orders. Furthermore, the $0-0$ vibrational band is formed higher in the atmosphere and thus more subject to any errors in the temperature distribution at high layers, which is where such are expected to occur as discussed in $\S$ 8.4. The region from 4318 to $4329 \AA$ was used instead.

We have no information about the $\mathrm{O}$ abundance in $\mathrm{HE}$ $2148-1247$, which is required to calculate the molecular equilibrium due to the potential importance of $\mathrm{CO}$. On the basis of the characteristics of other heavily C-enhanced metal-poor stars (see, e.g., Lucatello et al. 2003), we adopt $[\mathrm{O} / \mathrm{Fe}]=+0.5$ dex here and throughout this paper.

A synthesis of the region 4218-4329 $\AA$ superposed on the observed spectrum of HE 2148-1247 is shown in Figure 3. A La II line $4322.5 \AA$ occurs just blueward of the wavelength where strong $\mathrm{CH}$ absorption commences. The strength of this La II line cannot be reproduced without enhancing the La abundance by a factor comparable to that deduced from the more isolated lines of this species included in Table 1. The resulting $\mathrm{C}$ abundance is high, $\log [\epsilon(\mathrm{C})]=8.08 \pm 0.2$ dex, just 0.5 dex below the solar value.

The ratio of ${ }^{12} \mathrm{C} /{ }^{13} \mathrm{C}$ was determined by synthesizing selected regions between 4210 and $4225 \AA$. The wavelengths of the ${ }^{13} \mathrm{CH}$ lines given by Jorgensen (1994) appear to be correct in almost all cases examined in detail.

\footnotetext{
11 See http://cfa-www.harvard.edu/amdata/ampdata/amdata.shtml

12 See ftp://ftp.noao.edu.fts/visatl/README.
}

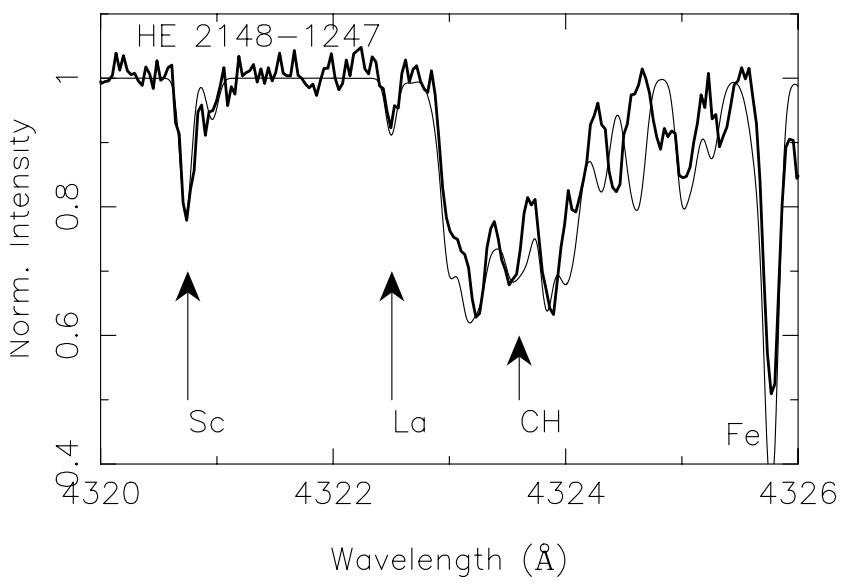

FIG. 3.-Synthesis of the region of the CH band from 4320 to $4326 \AA$. The abundances used are those from Table 3 . Note the good fit of the La II line at $4322.5 \AA$.

Figure 4 shows a spectral synthesis superposed on the observed spectrum of HE 2148-1247 for various regions with isolated ${ }^{13} \mathrm{CH}$ lines in the range $4210-4225 \AA$. Three different isotope ratios are shown, ${ }^{12} \mathrm{C} /{ }^{13} \mathrm{C}=3,10$, and 30 . Examining these features, we deduce a ratio of ${ }^{12} \mathrm{C} /{ }^{13} \mathrm{C}$ for HE 2148-1247 of $\sim 10$. A ratio smaller than 5 can be ruled out.

\subsection{Nitrogen}

The $\mathrm{N}$ abundance was determined from the synthesis of a region around the $0-0$ band head of the $\mathrm{CN}$ violet system shown in Figure 5. The $\mathrm{CN}$ line list was from Kurucz (1994), to which we added the $\mathrm{CH}$ lines in that region, as well as the strongest atomic lines, which are from $\mathrm{Fe}$ I and

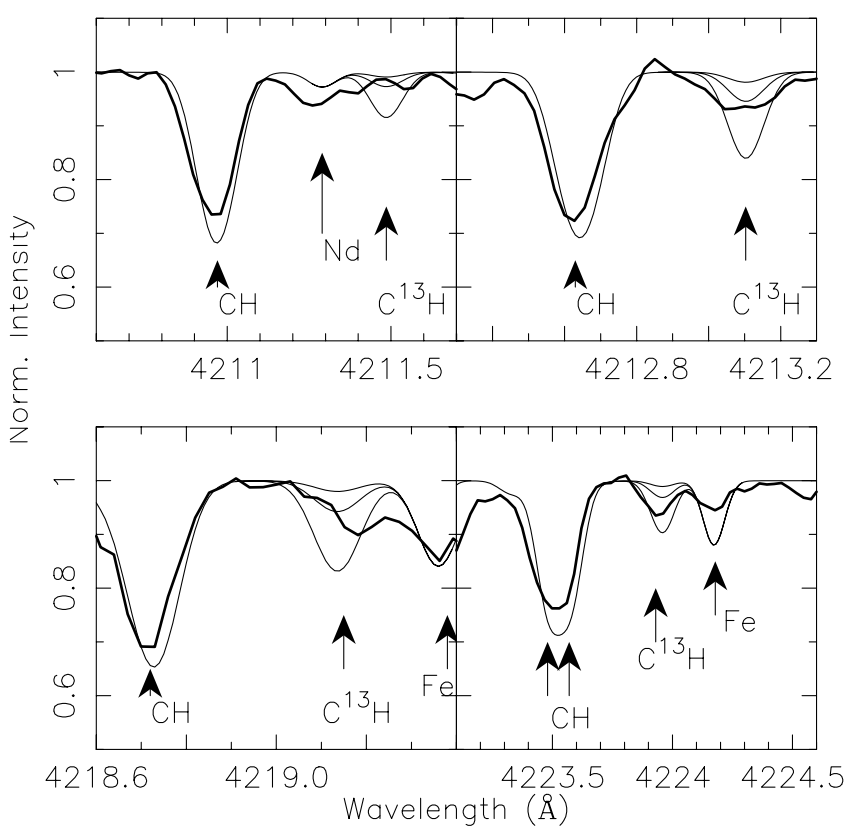

FIG. 4.-Synthesis of selected regions with relatively isolated features of ${ }^{13} \mathrm{CH}$ overlaid on the spectrum of HE $2148-1247$. Three different isotope ratios are shown, ${ }^{12} \mathrm{C} /{ }^{13} \mathrm{C}=3,10$, and 30 . The abundances used are those from Table 3. The spectrum in the lower left panel is the sum of two adjacent orders. It has not been smoothed. The spectra shown in the other three panels have been slightly smoothed. 


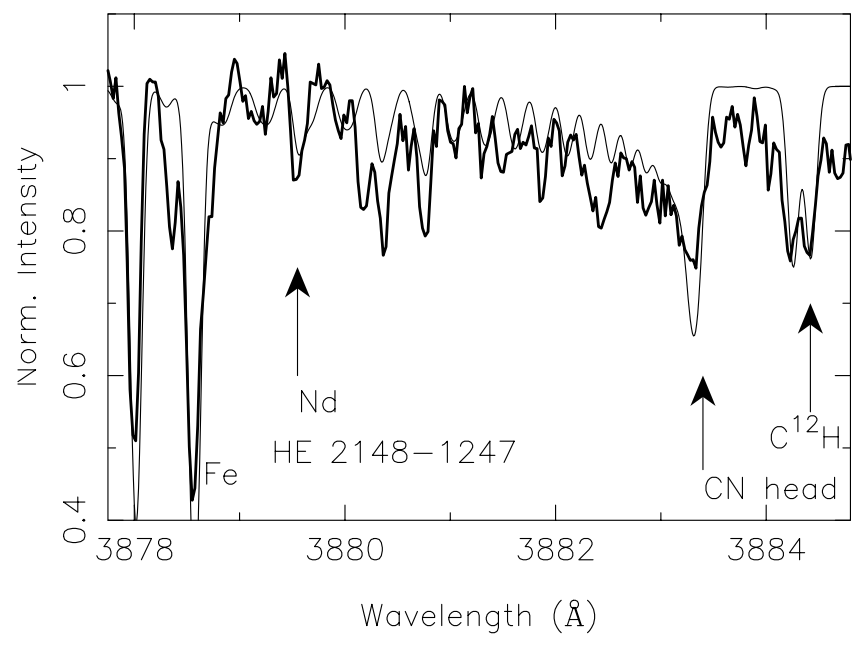

FIG. 5.- Synthesis of the region of the band head of the $0-0$ violet $\mathrm{CN}$ band is shown. The abundances used are those from Table 3 . The wing of the Balmer line $\mathrm{H} 8$ at $3889.05 \mathrm{~A}$ lowers the apparent continuum at the red end of the region shown.

Cr I. We also found we had to add several features of $\mathrm{Nd}$ II. The high $\mathrm{C}$ abundance derived from the syntheses of the $\mathrm{CH}$ features described above was used as an input into the $\mathrm{CN}$ synthesis, as was the ratio derived for ${ }^{12} \mathrm{C} /{ }^{13} \mathrm{C}$. This high $\mathrm{C}$ abundance is, in fact, required to fit the two strong $\mathrm{CH}$ lines at 3884.2 and $3884.4 \mathrm{~A}$, just redward of the $\mathrm{CN}$ band head. A high $\mathrm{N}$ abundance is derived from this synthesis of the $0-0$ violet $\mathrm{CN}$ band, whose exact value depends on the choice of $\mathrm{C}$. To first order, for small changes in $\mathrm{C}$, the required value of $\mathrm{N}$ can be obtained by assuming that the product of the $\mathrm{C}$ and $\mathrm{N}$ abundances is constant.

\subsection{Lead and Thorium}

The only $\mathrm{Pb}$ I line that is accessible within the wavelength range of the spectra is at $4057.8 \AA$. This line is extremely strong in the spectrum of HE 2148-1247. While abundances based on a single line may be considered suspicious, in this case the presence of this single strong line of $\mathrm{Pb}$ is confirmed beyond any doubt as it was also easily detected in the spectrum taken in 2002 September as part of the radial velocity monitoring campaign. As shown in Figure 6, the Mg I line at $4057.5 \AA$ is a weak feature in the blue wing of the much stronger $\mathrm{Pb}$ I line. A spectral synthesis is not really necessary to establish the $\mathrm{Pb}$ abundance, but it was used to demonstrate that the $\mathrm{CH}$ lines in this region from $B^{2} \Sigma^{-}-X^{2} \Pi$ transition, while definitely present, are not playing a significant role, even with the enhanced $\mathrm{C}$ abundance derived for HE 2148-1247. The synthesis was carried out using both the hyperfine and isotopic splittings of Simons et al. (1989) assuming a solar distribution among the four stable isotopes of $\mathrm{Pb}$ and also using a single line to represent this $\mathrm{Pb}$ feature. There is little difference in the resulting $\mathrm{Pb}$ abundance, $\epsilon(\mathrm{Pb})=+2.8 \pm 0.2$ dex. This is an extremely high abundance of lead, well above that of the Sun $[\epsilon(\mathrm{Pb})=2.0]$ and among the highest known for any metal-poor star.

The strongest and cleanest accessible line of thorium in this wavelength region is the $4019.139 \AA$ line of Th II. We adopt $\log (g f)=-0.27$ dex from Lawler, Whaling, \& Grevesse (1990) for this Th II line but do not adopt their solar Th abundance. This line is blended with a Co I line at

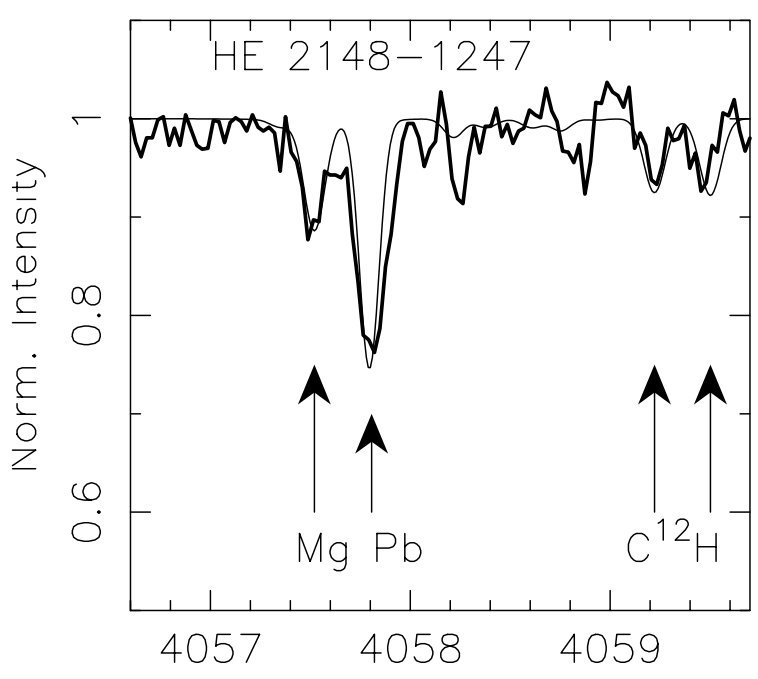

Wavelength $(\AA)$

FIG. 6.-Spectral synthesis of the region of the $\mathrm{Pb}$ I line at $4057 \AA$ is shown. The observed spectrum of HE $2148-1247$ is shown by the heavy solid curve, while that of the synthesis is indicated by the thin curve. The lead abundances used is $\log [\epsilon(\mathrm{Pb})]=+2.65$ dex.

$4019.125 \AA$ and with a ${ }^{13} \mathrm{CH}$ line at $4019.136 \AA$. The Ce II line at $4019.047 \AA$, which must be included because of the enhanced abundances of the rare earths in HE 2148-1247, also contributes to the blue wing of the blend. (See Morrell, Källander, \& Butcher 1992 for details; Johnson \& Bolte 2001 give an up-to-date line list for the region of the Th II 4019 A line.)

Figure 7 shows the synthesis, which used $\log [\epsilon(\mathrm{Th})]=$ $-1.4,-0.5$, and -0.2 dex. As expected, a high enhancement of $\mathrm{C}$ is required to produce an adequate fit to the pair of ${ }^{12} \mathrm{CH}$ lines at $4020.1 \AA$. We infer is a very high Th abundance $\log [\epsilon(\mathrm{Th})]=-0.55$ dex. However, the dominant contributor to the blend observed in HE 2148-1247 at 4019.1 $\AA$ is ${ }^{13} \mathrm{CH}$ instead of $\mathrm{Th}$, and any error in the ${ }^{12} \mathrm{C} /{ }^{13} \mathrm{C}$ ratio

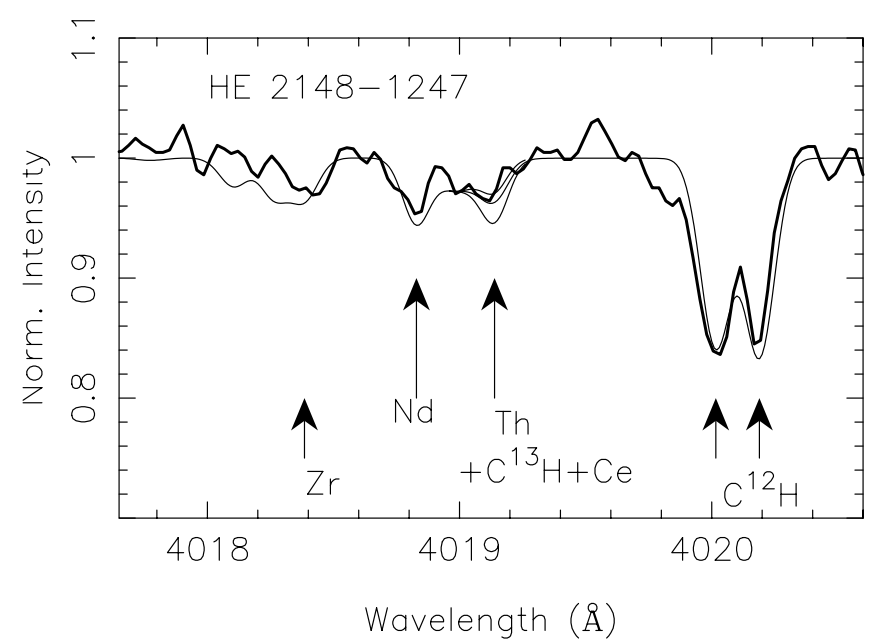

FIG. 7.-Slightly smoothed observed spectrum of HE 2148-1247 (thick line) is overlaid by the synthesis of the region around the Th II line at $4019 \AA$ (thin line); $\epsilon(\mathrm{Th})=-1.4,-0.5$, and -0.2 dex for the three cases shown. The abundances utilized for other elements in the synthesis are set to the values described in Table 3. 
will strongly affect the derived $\mathrm{Th}$ abundance. If ${ }^{12} \mathrm{C} /{ }^{13} \mathrm{C}$ is less than the value of 10 adopted in this synthesis, the $\mathrm{Th}$ contribution to the total observed feature at $4019 \AA$ would become very small, and thus the deduced Th abundance lowered by a large factor. The synthesis shows that a line at $4019.15 \AA$ is present in the spectrum of HE 2148-1247; however, because of the overlapping blends, the detection of Th is not yet secure. This will require even higher precision high-resolution spectra. This is unfortunate because a secure detection of Th would make a large $r$-process contribution mandatory. The spectrum at the wavelength of the strongest line of U II accessible to us at $3859.57 \AA$ does not have enough precision to provide an interesting limit on the U abundance.

\subsection{The Effect of the $\mathrm{C}$ Excess on Model Atmospheres}

Carbon and to a lesser extent nitrogen are highly enhanced in HE 2148-1247, with $[\mathrm{C} / \mathrm{Fe}]=+1.9$ dex. Could such a large enhancement of carbon produce excess absorption of the continuum through various molecular bands, which would affect the temperature profile in the atmosphere and hence the abundances we derived? We first note that such effects will be larger for individual abundances [i.e., $\epsilon(\mathrm{X})]$ than for the relative abundances of two species. Hill et al. (2000) compare the $T$ - $\tau$ profiles for three model atmospheres, one of which properly includes the $\mathrm{C}$ and $\mathrm{N}$ enhancements for the stars they analyze, while another is taken from the Kurucz grid used here. The largest difference between the two above $\tau(5000 \AA)=1$ is about $200 \mathrm{~K}$. However, the stars they consider, while having similar $\mathrm{C}$ and $\mathrm{N}$ enhancements to HE 2148-1247, are cool giants with $T_{\text {eff }} \sim 4800 \mathrm{~K}$, much lower than that of HE 2148-1247. As shown by Lucatello et al. (2003), the total absorption in the $\mathrm{CH}$ and $\mathrm{CN}$ bands for a star similar in $[\mathrm{Fe} / \mathrm{H}]$ and in [C, N/Fe] to HE 2148-1247 is not significant when compared to the total flux, approximated by a blackbody, provided that oxygen is not significantly enhanced as well.

Strongly enhanced $\mathrm{C}$, especially when accompanied by highly enhanced $\mathrm{N}$, does have a significant effect on the ability to detect features from certain elements whose crucial lines fall within the wavelength regime covered by the $\mathrm{CH}$ or $\mathrm{CN}$ bands. The $\mathrm{Pb}$ I line at $4057 \AA \mathrm{A}^{13}$ the Th II line at $4019 \AA$, and the blue Eu II lines fall into this category. It is not always feasible to obtain red spectra from which to pick up the $6645 \AA$ line of Eu II, and there are no suitable red lines of any ion of $\mathrm{Pb}$ or Th. In this context, recall that HE 2148-1247 is a main-sequence turnoff region star, with much weaker lines and molecular bands because of its higher $T_{\text {eff }}$.

\section{ALTERNATIVE EXPLANATIONS}

We reject the notion that the abundance peculiarities seen in HE 2148-1247 did not originate from a nuclear process but instead some type of radiative levitation. Some classes of Ap stars display enormous enhancements of the rare earths, the most extreme known example of which is HD 101065, Przybylski's star, where the rare earths are more or less uniformly enhanced over their solar abundances by a factor of $\gtrsim 10^{3}$. Radiation pressure, primarily due to

\footnotetext{
13 This line is the reddest of the expected strong $\mathrm{Pb}$ I lines. Detection of any of the bluer $\mathrm{Pb}$ I lines will be even more difficult because of crowding of lines in the spectrum.
}

absorption lines, can support an atom only in a very stable atmosphere, and the effect is highly dependent on luminosity. Among old and metal-poor populations, this is seen only in hot horizontal branch stars (see Behr, Cohen, \& McCarthy 2000 and references therein). The distribution of the resulting abundances is that elements with low true abundances rise and appear enhanced, while those with high initial abundances, particularly those with few absorption lines, sink and are depleted. The detailed analysis of Przybylski's star by Cowley et al. (2000) yields an abundance distribution for the Fe peak elements that is not consistent with that typical of metal-poor stars and seen in HE $2148-1247$.

Furthermore, as discussed in $\S 10.1$, the abundance peculiarities of HE 2148-1247 are shared by several other metal-poor stars that are cool giants or subgiants whose atmospheres are kept well mixed by convection. Radiative levitation is not possible in such stars.

\section{DISCUSSION OF ABUNDANCES IN HE 2148-1247}

The elemental abundances determined for HE 21481247 are critical for understanding the sites for the $s$ - and $r$-processes of nucleosynthesis. The relevant observational facts for this star with a low value of $[\mathrm{Fe} / \mathrm{H}] \approx-2.3$ are as follows:

1. The abundances of the elements from $\mathrm{Mg}$ to $\mathrm{Ni}$ including Fe relative to hydrogen are far below the solar values and approximately follow the yield patterns inferred for very massive stars and some Type II supernovae [SNe II $(L)$ by Qian \& Wasserburg (2002).

2. In general, the abundances of the elements from $\mathrm{Ba}$ to Dy relative to hydrogen are remarkably close to the solar values.

3. Th may be present in high abundance.

4. The elements $\mathrm{Sm}, \mathrm{Eu}, \mathrm{Gd}$, and Dy closely follow the solar $r$-pattern.

5. The elements $\mathrm{Ba}, \mathrm{La}, \mathrm{Ce}, \mathrm{Pr}$, and $\mathrm{Nd}$ approximately follow the solar main $s$-pattern, demonstrating great $s$ process enrichment

6. The $\mathrm{Ba} / \mathrm{Eu}$ ratio is $\sim 10$ times that for the solar $r$ pattern.

7. The $\mathrm{Pb} / \mathrm{Ba}$ ratio is $\sim 10$ times that for the solar main $s$-pattern.

In addition, $\mathrm{C}$ and $\mathrm{N}$ are highly enhanced in $\mathrm{HE}$ $2148-1247$. Figure 8 displays the distribution of the abundances for the 27 elements obtained here. It can be seen that, except for $\mathrm{C}$ and $\mathrm{N}$, almost all elements below $Z=40(\mathrm{Zr})$ are very low compared to solar abundances. Above $Z=40$ they appear to be rather close to solar abundances. Figure 9 focuses on the region between $\mathrm{Sr}$ and $\mathrm{Th}$. The solid line segments indicate the total solar abundance for the same set of elements. ${ }^{14}$ It can be seen that $\mathrm{Eu}$ and $\mathrm{Sm}$ are distinctly below the solar curve.

If we assume that HE 2148-1247 was initially formed of metal-poor gas with an elemental abundance distribution characteristic of its present $[\mathrm{Fe} / \mathrm{H}]$, then it has large enhancements of $\mathrm{C}$ and $\mathrm{N}$ and of the heavy elements. Figure 10 shows the abundance distribution from $\mathrm{Sr}$ to $\mathrm{Th}$ in $\mathrm{HE}$ $2148-1247$ as in Figure 9 but with the $r$ - and $s$-process solar

\footnotetext{
14 The solar line also includes the abundances of $\mathrm{V}$ and $\mathrm{Tb}$ for clarity.
} 


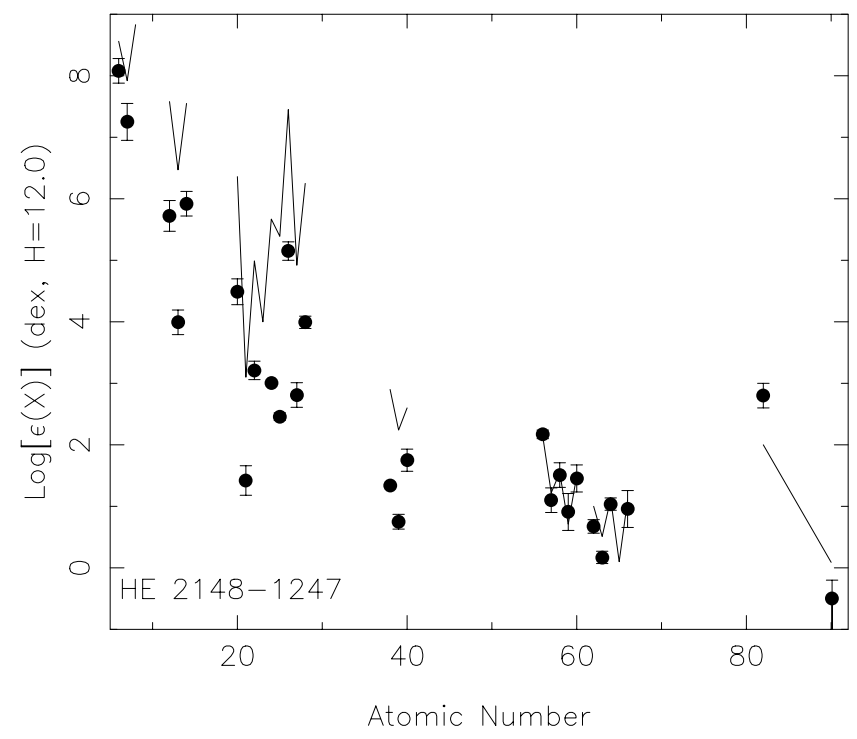

FIG. 8.-Abundances of 27 elements in HE 2148-1247 (see Table 3) are shown as a function of atomic number, with appropriate error bars. The total solar abundances for these elements are indicated by the line segments.

abundance distributions, taken from Burris et al. (2000), following Käppeler et al. (1989), indicated by solid and dashed lines. In both cases, these are normalized to match the abundance of Ba in HE 2148-1247. Figure 11 is similar to Figure 10, but in this case the $r$ - and $s$-process solar abundance distributions are normalized to Eu.

Examination of these two figures demonstrates clearly that HE 2148-1247 shows large contributions from both the $s$ - and the $r$-processes. Neither component alone is capable of matching the observed heavy element abundances seen. We note that the ratios of Ba to Eu in the solar $r$ process, $s$-process, and total solar inventory (adopted from Burris et al. 2000) are 8.9, 1860, and 59, respectively, while

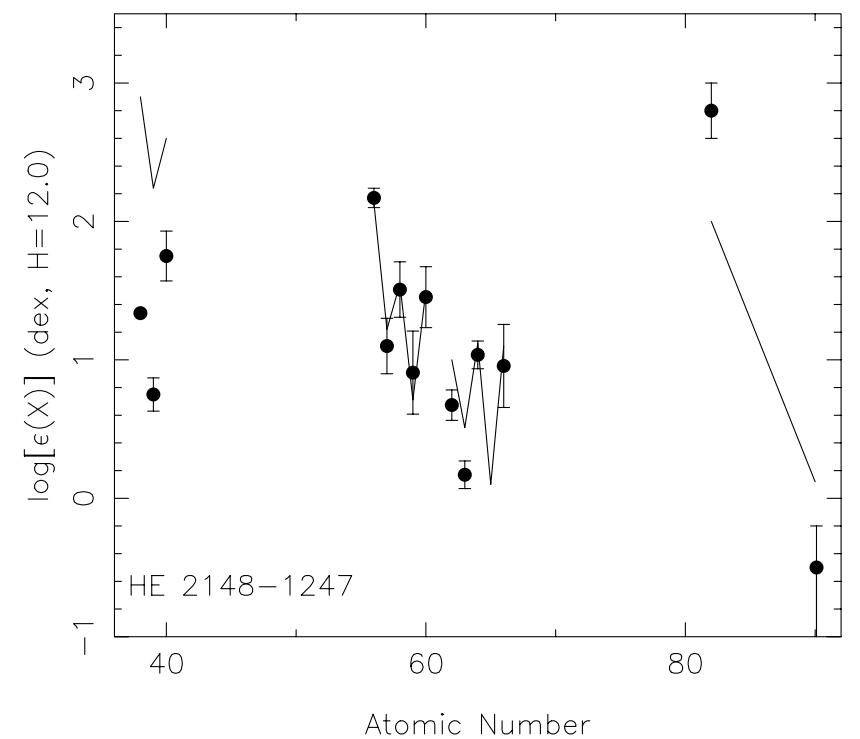

FIG. 9.-Abundances of the heavy elements in HE 2148-1247 are shown as a function of atomic number, with appropriate error bars. The total solar abundances for these elements are indicated by the line segments.

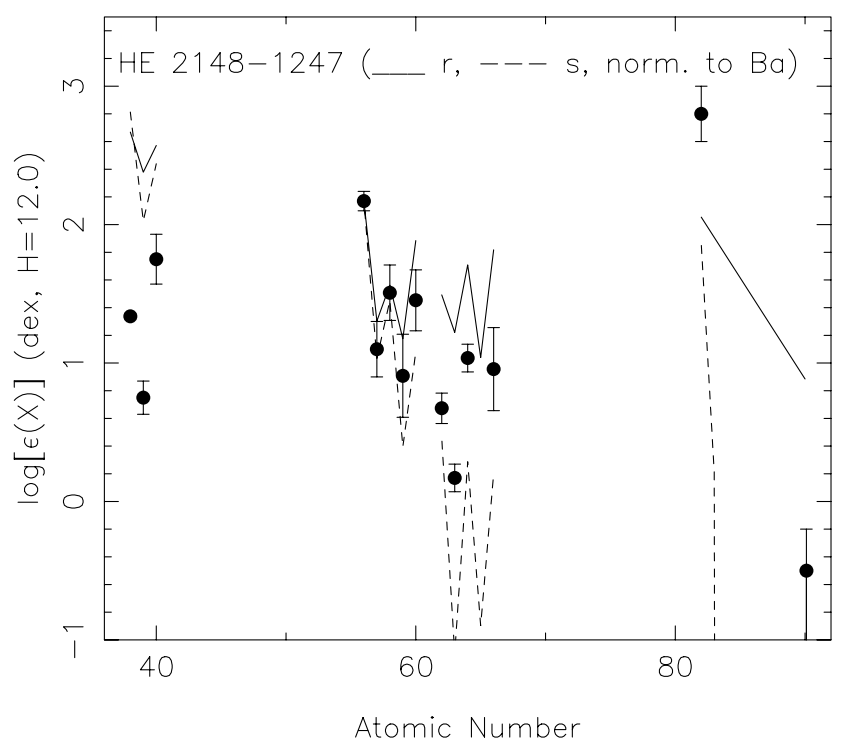

FIG. 10.-Derived abundances for HE 2148-1247 for the heavy elements are shown as a function of atomic number, with appropriate error bars. The $r$ - and $s$-process solar abundances from Burris et al. (2000), scaled to match those of HE 2148-1247 at Ba in each case, are shown as the solid and dashed line segments, respectively.

that of HE $2148-1247$ is $\sim 100$. A comparison with $\mathrm{La}$ avoids the difficulties introduced into the abundance analysis by the very strong Ba II lines in HE 2148-1247. The ratio of La to Eu in the solar $r$-process, $s$-process, and total solar inventory (again adopted from Burris et al. 2000) is 1.2, 136, and 4.8, respectively, while that of HE $2148-1247$ is $~ 8.5$. We have thus attempted to reproduce the abundance pattern seen in HE 2148-1247 with a sum of contributions from the two neutron capture processes. We find that adding $62 \%$ of the total solar $r$-process inventory to $83 \%$ of the total solar $s$-process inventory reproduces the abundances of all the elements observed in HE 2148-1247 over the

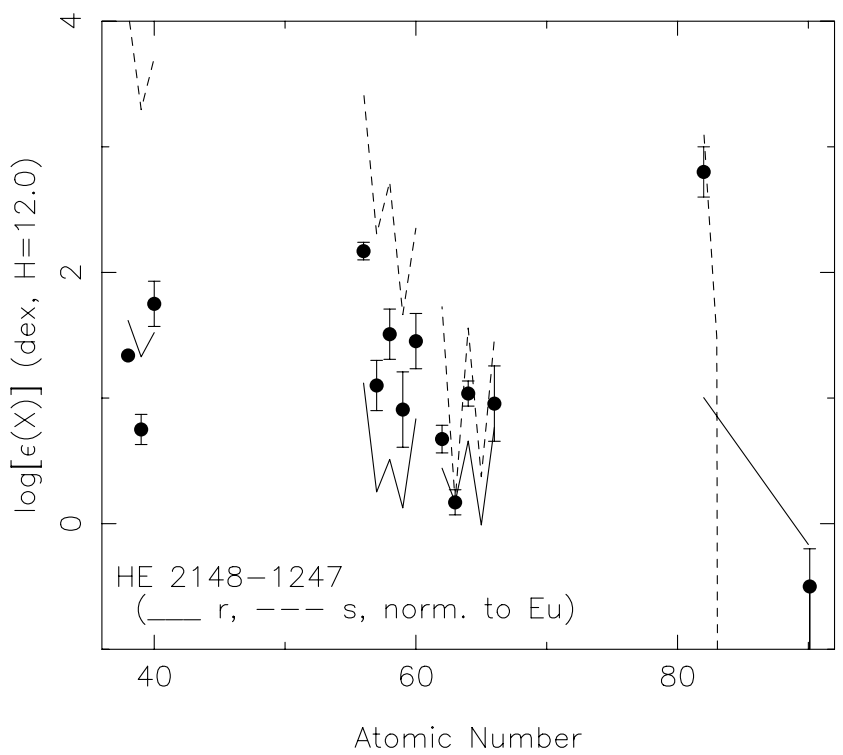

Fig. 11.-Same as Fig. 10, but with the $r$-and $s$-process solar abundances from Burris et al. (2000) scaled to match those of HE 2148-1247 at Eu in each case. 
TABLE 5

Comparison of the Observed and Predicted Abundances in HE 2148-1247 Using the SOLAR $r$ - AND $s$-PROCESS INVENTORY

\begin{tabular}{|c|c|c|c|c|}
\hline Element & $\begin{array}{c}\epsilon(\mathrm{X}) \\
\left(\mathrm{H}=10^{12}\right)\end{array}$ & $\begin{array}{l}\text { Predicted } \epsilon(\mathrm{X}-r)^{\mathrm{a}} \\
\quad\left(\mathrm{H}=10^{12}\right)\end{array}$ & $\begin{array}{c}\text { Predicted } \epsilon(\mathrm{X}-s)^{\mathrm{b}} \\
\quad\left(\mathrm{H}=10^{12}\right)\end{array}$ & $\begin{array}{l}\Delta \text { (Pred.-Obs. }) \\
\quad(\text { dex })\end{array}$ \\
\hline $38(\mathrm{Sr}) \ldots \ldots \ldots \ldots \ldots$ & 21.78 & 56.10 & 603.72 & 1.48 \\
\hline $39(Y) \ldots \ldots \ldots \ldots$ & 5.62 & 28.82 & 98.36 & 1.35 \\
\hline $40(\mathrm{Zr}) \ldots \ldots \ldots \ldots$ & 56.23 & 44.88 & 255.03 & 0.73 \\
\hline 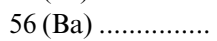 & 147.91 & 17.82 & 137.24 & 0.02 \\
\hline $57(\mathrm{La})$ & 12.59 & 2.42 & 10.01 & -0.01 \\
\hline $58(\mathrm{Ce})$ & 32.21 & 4.40 & 26.21 & -0.02 \\
\hline 59 (Pr) & 8.09 & 1.80 & 2.30 & -0.30 \\
\hline $60(\mathrm{Nd}) \ldots \ldots \ldots \ldots \ldots$ & 28.38 & 9.24 & 11.19 & -0.14 \\
\hline $62(\mathrm{Sm}) \ldots \ldots \ldots \ldots \ldots$ & 4.71 & 3.74 & 2.65 & 0.13 \\
\hline $63(\mathrm{Eu})$ & 1.48 & 2.00 & 0.07 & 0.15 \\
\hline $64(\mathrm{Gd}) \ldots \ldots \ldots \ldots \ldots$ & 10.86 & 6.17 & 1.80 & -0.14 \\
\hline 66 (Dy).................. & 9.04 & 7.92 & 1.47 & 0.02 \\
\hline $82(\mathrm{~Pb}) \ldots \ldots \ldots \ldots$ & 630.96 & 13.64 & 67.73 & -0.89 \\
\hline $90(\mathrm{Th}) \ldots \ldots \ldots \ldots$ & 0.32 & 0.92 & & 0.47 \\
\hline
\end{tabular}

a The $r$-process contribution in HE 2148-1247 is set as 0.62 times the solar $r$-process inventory given by Burris et al. 2000 in the same units.

b The $s$-process contribution in HE $2148-1247$ is set as the 0.83 times the solar $s$-process inventory given by Burris et al. 2000 in the same units.

range of atomic number from 56 to 66 to within the observational errors, as is shown in Table 5. Thus, HE 2148-1247, which is, as judged by the elements $\mathrm{Na}$ through $\mathrm{Zr}$, very metal-poor, has almost the total solar inventory of heavy neutron capture elements. However, Table 5 shows that the observed abundances of $\mathrm{Sr}, \mathrm{Y}$, and $\mathrm{Zr}$ are far $(4-10 \sigma)$ below those predicted by this two-component sum. Furthermore, the $\mathrm{Pb}$ abundance is too high by more than $4 \sigma$, while the $\mathrm{Th}$ abundance, which has a large observational error, is $\geq 1.3 \sigma$ smaller than the fit.

\subsection{Comparison with Other Stars}

On the basis of examination of the many spectra of very metal-poor stars, we confirm the result of Norris, Ryan, \& Beers (1997) that enhancement of $\mathrm{C}$ is common among very metal-poor stars. Large enhancement of $\mathrm{N}$ is somewhat rarer but not unusual. We defer discussion of the $\mathrm{C}$ and $\mathrm{N}$ abundances to $\S 10.2$.

Our experience to date suggests that enhancement of the heavy elements is rare among metal-poor stars, and large enhancements are very rare. Previous examples of $r$ process-enhanced very metal-poor stars that show no sign of $s$-process enhancement have been found by several groups. The most extreme such stars known are CS 22892-052 (Sneden et al. 1994, 1996, 2000) and CS 31082-001 (Cayrel et al. 2001; Hill et al. 2002), which have $\log [\epsilon(\mathrm{Eu})]$ of -0.9 and -0.75 dex, respectively, with $[\mathrm{Fe} / \mathrm{H}]=-3.1$ and -2.9 dex, respectively; $\log [\epsilon(\mathrm{Eu})]$ in $\mathrm{HE}$ $2148-1247$ is much larger, +0.17 dex. In addition to these two very well-studied extremely $r$-process-enhanced stars, a number of less extreme such stars have been analyzed by Burris et al. (2000), Johnson \& Bolte (2001), and Cowan et al. (2002). Among them, the bright star BD $+17^{\circ} 3248$ is of particular interest, because very high precision spectra for this star can easily be obtained with Hubble Space Telescope and from the ground. Many $r$-process elements, not easily seen under normal conditions, can thus be detected in the spectrum of this star (Cowan et al. 2002).
Similarly, there are a number of very metal-poor stars known to show large enhancements of $s$-process material. There is a sample of eight such stars in Aoki et al. (2001). We have also found another such star in the HES, HE 0024-2523 (Lucatello et al. 2003), which shows many similarities to HE 2148-1247, is known to be a binary, but lacks any sign of an $r$-process excess.

Figure 12 compares the abundance distribution in $\mathrm{HE}$ $2148-1247$ to that of the extreme $r$-process star CS 31082-001 (data from Hill et al. 2002) and to the s-processdominated star HD 196944 (data from Van Eck et al. 2001, Aoki et al. 2001, and Zacs, Nissen, \& Schuster 1998)..$^{15}$ The effect of the $s$-process enhancement in the abundance pattern in HE 2148-1247 is quite obvious in the upper panel of this figure; the enhancement of $\mathrm{Ba}$ in the difference between the abundances in HE 2148-1247 and in CS 31082-001 is much larger than that of $\mathrm{Eu}$. On the other hand, the enhancement of Eu in HE 2148-1247 relative to that of the $s$-process star is apparent in the bottom panel and indicates a strong $r$-process contribution to the total inventory in $\mathrm{HE}$ $2148-1247$.

HE 2148-1247 is perhaps the best-studied example of a small group of metal-poor stars displaying signs of both $s$ and $r$-process enhancement. Hill et al. (2000) have studied two additional such stars from the HK Survey. We attempt to quantify the $r$ - and $s$-process contributions in a large sample of metal-poor stars using the Eu abundance to indicate the former and the $\mathrm{Ba}$ abundance to indicate the latter. We selected a sample of low-metallicity $([\mathrm{Fe} / \mathrm{H}]<-2.0$ dex $)$ stars from the literature, all of which show signs of enhanced abundances of the neutron capture elements; all, except one, have Eu detections. In addition to the three extremely $r$-process stars noted above, we include HD 115444, with data from Johnson \& Bolte (2001) and from Westin et al. (2000). Less heavily $r$-process-enhanced stars were taken from Johnson \& Bolte (2001). Additional stars were taken

${ }^{15} \mathrm{~A}+0.6$ dex correction for non-LTE in $\mathrm{Al}$ has been applied throughout. 

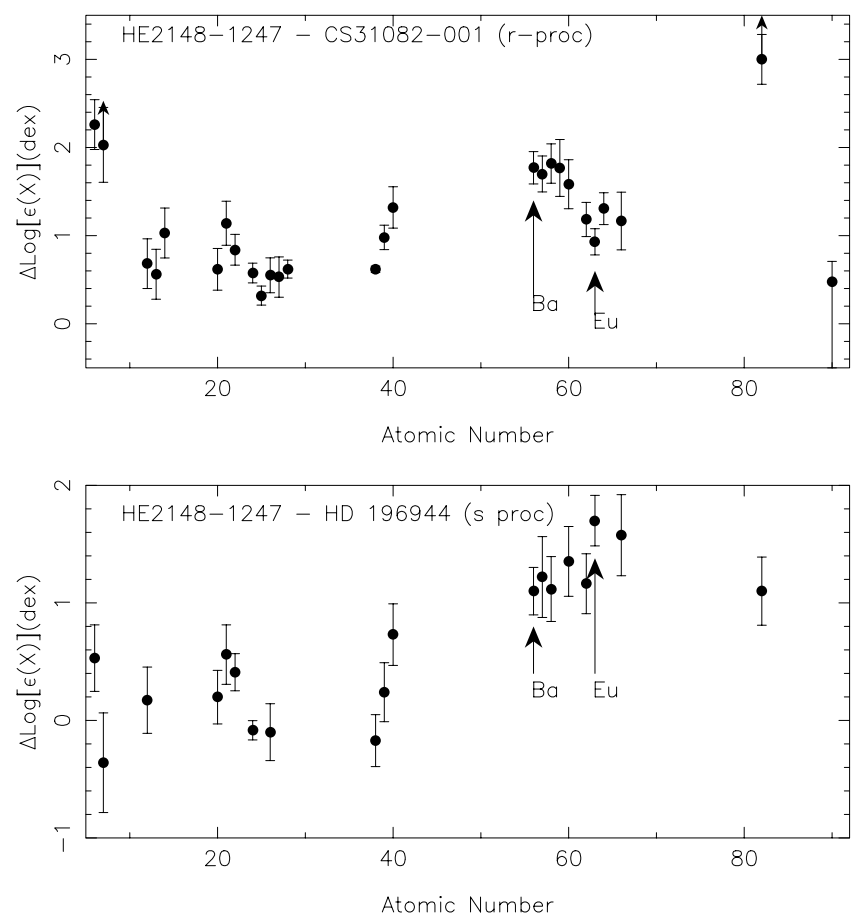

FIG. 12.-Abundances in HE 2148-1247 are compared to those of the $r$-process star CS 31082-001 and to the s-process-dominated star HD 196944. Only upper limits for $\mathrm{N}$ and for $\mathrm{Pb}$ are available for CS $31082-001$. The difference in $\log (\epsilon)$ between the two stars is plotted for each element in common.

from Hill et al. (2000), Norris et al. (1997), and Aoki et al. (2002a). The $r$ - and $s$-process content of each star are plotted in Figure 13. The four known stars with highly enhanced $r$ process abundances are plotted as the large stars in the figure. They are plotted at both their nominal abundances (large symbols) and then with $\epsilon(\mathrm{Ba})+1 \sigma$ and $\epsilon(\mathrm{Eu})-1 \sigma$ error, in order to maximize the possible $s$-process contribution (Fig. 13, open triangles). HE 2148-1247 is shown by the large filled circle, while HE 0024-2523, which only has an upper limit for $\mathrm{Eu}$, is shown by the slightly smaller filled circle. The solid line denotes the $\mathrm{Ba} / \mathrm{Eu}$ ratio expected for pure $r$-process material, the dashed line for pure $s$-process material, and the dot-dashed line for material with the solar ratio of $\mathrm{Ba}$ to $\mathrm{Eu}$. The position of the Sun is marked by the letter "S."

There are two key points here. The first is the well-known wide range of $r / s$ ratio, i.e., the existence of very metal-poor stars covering the range from those with neutron capture elements almost purely produced via the $r$-process to those heavily dominated by the $s$-process.

The second is the magnitude of the contributions. Note that Figure 13 is plotted with a logarithmic scale for both axes. All the stars shown in the figure are very metal-poor, and yet in a few of them, including HE 2148-1247, we see almost the total solar inventory of both $r$-and $s$-process elements. On the other hand, the "pure" $r$-process stars do not reach more than $10 \%$ of the solar Eu abundance, nor more than about $1 \%$ at most of the solar Ba abundance.

\subsection{Implications for the Site of the Nucleosynthesis}

The enhancement of the $r$-process elements in HE $2148-1247$ as compared to the Fe peak elements is very large, comparable to or larger than that of the most

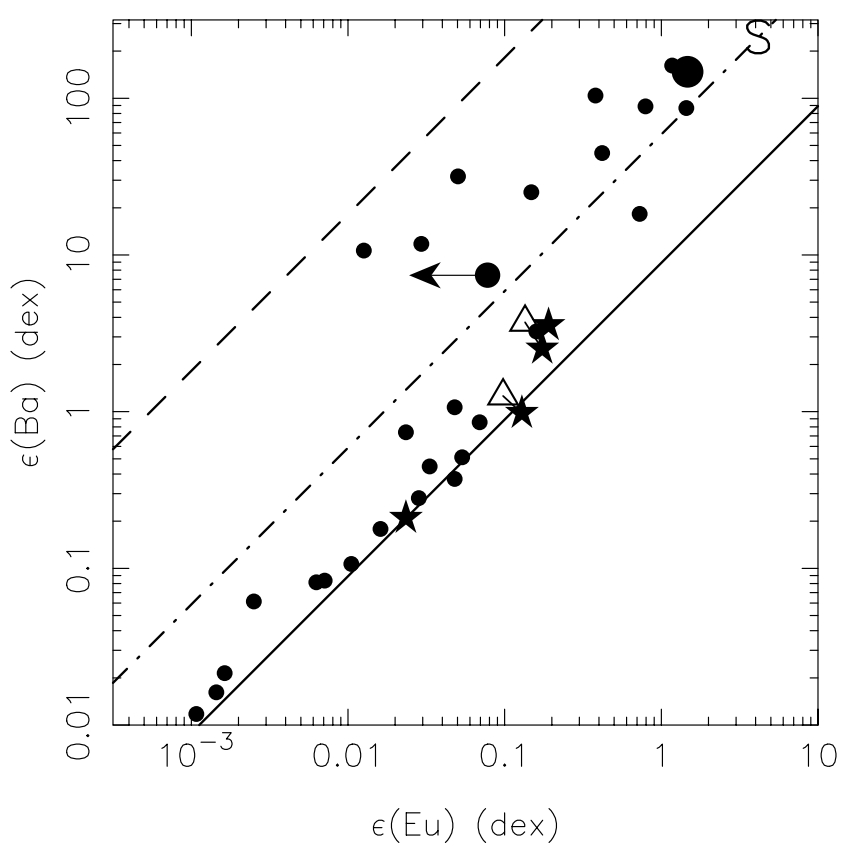

FIG. 13.- Ratios of Eu (representing the $r$-process) to $\mathrm{Ba}$ (representing the $s$-process) are shown for a sample of metal-poor stars, all with $[\mathrm{Fe} / \mathrm{H}]$ $<-2.0$ dex. The sources for the abundances are given in the text. The large stars denote the four highly enhanced $r$-process stars with their nominal abundances. In two cases, the results are shown for the abundances including the errors so as to maximize the resulting $s$-process contribution. These are indicated by open triangles connected by a line to the symbol denoting the nominal abundance case. HE $2148-1247$ is shown as the large filled circle. HE 0024-2523 is shown as a slightly smaller filled circle with an arrow indicating only an upper limit for $\epsilon(\mathrm{Eu})$. The solid line denotes the $r$-process $\mathrm{Ba} / \mathrm{Eu}$ ratio, the dashed line denotes that for the $s$-process, and the dot-dashed line denotes the solar $\mathrm{Ba} / \mathrm{Eu}$ ratio. The letter " $\mathrm{S}$ " indicates the position of the Sun.

enhanced pure $r$-process star discovered to date. Similarly the enhancement of the $s$-process elements is also very large and again comparable to the most extreme known metalpoor stars in mass transfer binaries. The enhancement of $\mathrm{C}$ and $\mathrm{N}$ is also very large. Furthermore, the star appears to be a long-period binary.

All scenarios for producing $\mathrm{C}$ in low-mass stars, whether via the classical dredge-up on the asymptotic giant branch (AGB) or whether invoking the path to mixing at the $\mathrm{He}$ flash for very metal-poor stars recently predicted by Fujimoto, Ikeda, \& Iben (2000), require that the star be at least as evolved to incur the He flash. But HE 2148-1247 is a star near the main-sequence turnoff and hence cannot have produced any of the anomalies we see by itself.

McClure (1983) discovered that the $\mathrm{CH}$ stars are all single-line spectroscopic binaries. Their spectral peculiarities, including the presence of strong lines of some of the $s$ process elements, have been attributed to mass transfer between the components of a binary system. If a very metalpoor star has as its binary companion a more massive star that has already evolved to or through the AGB phase, enhancements of $\mathrm{C}, \mathrm{N}$, and the $s$-process elements can be produced (see, e.g., Jorissen \& Boffin 1992; Han et al. 1995). A binary star model places constraints on the binary separation and period, as the mass transfer must not occur while the primary is on the RGB instead of the AGB, and too wide a separation may make the mass transfer inefficient, as discussed by Jorissen \& Boffin (1992). 
Goriely \& Siess (2001) and Siess, Livio, \& Lattanzio (2002) discuss the nucleosynthesis of $s$-elements in lowmetallicity AGB stars and find that even without Fe seed nuclei, extensive $s$-process production may occur. Since HE $2148-1247$ is a binary, the extremely enhanced C and N and $s$-process elements can be explained following the mass transfer scenario outlined above. The $s$-process in very metal-poor stars may produce copious amounts of $\mathrm{Pb}$ if there is a high neutron to seed ratio (Busso et al. 2001; Goriely \& Siess 2001), which is also observed in HE 2148-1247 and in HE 0024-2523, another example of an $s$-process-enhanced dwarf star found in the course of the Keck Pilot Project (see Lucatello et al. 2003).

The existence of at least two dwarf stars with substantial $\mathrm{C}, \mathrm{N}$, and $s$-process enhancements suggests that even among the very metal-poor giant stars showing these phenomena, the probable cause is mass transfer in a binary rather than production via internal nucleosynthesis within the star itself. Recall that very metal-poor giants are assumed to be old and hence have masses $\sim 0.8 M_{\odot}$. Opportunities for internal nucleosynthesis and dredge-up in such stars are very limited.

Figure 14 illustrates the $s$-process enhancement parameterized as $[\mathrm{Ba} / \mathrm{Fe}]$ as a function of $[\mathrm{Fe} / \mathrm{H}]$ for the same sample of very metal-poor stars shown in Figure 13. Those stars with $\mathrm{Ba} / \mathrm{Eu}<25$ (i.e., those that are $r$-process-dominated) are shown as open circles, while those with $\mathrm{Ba} / \mathrm{Eu}>25$ are shown as filled circles. Recall that all these stars have $[\mathrm{Fe} / \mathrm{H}] \leq-2.0 \mathrm{dex}$. Note the very high $s$-process enhancement (a factor of 200 over that expected from the solar $s$ -

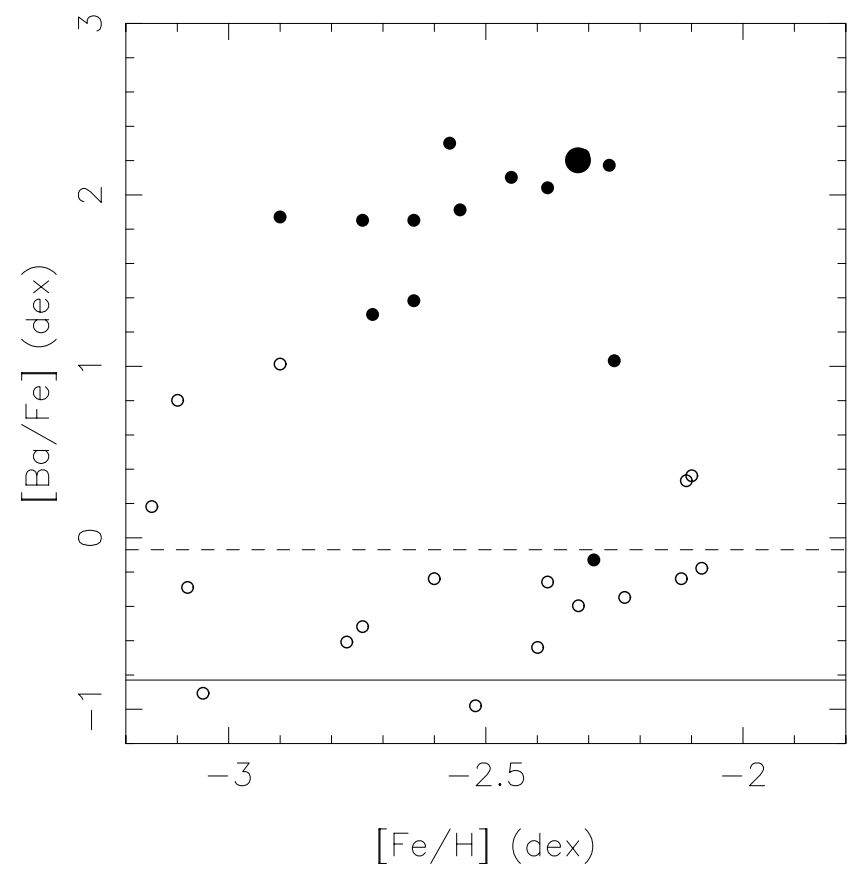

FIG. 14.-Enhancement of the $s$-process elements parameterized as $[\mathrm{Ba} / \mathrm{Fe}]$ over the nominal $s$-process inventory expected for the $[\mathrm{Fe} / \mathrm{H}]$ of the star is plotted as a function of $[\mathrm{Fe} / \mathrm{H}]$ for the sample of very metal-poor stars shown in Fig. 13. Those stars with $\mathrm{Ba} / \mathrm{Eu}<25$ (i.e., the $r$-dominated stars) are shown as open circles, while stars with $\mathrm{Ba} / \mathrm{Eu}>25$ (i.e., the $s$ dominated stars) are shown as filled circles. HE 2148-1247 is shown with a larger symbol. The solid horizontal line indicates the fraction of $\mathrm{Ba}$ in the Sun produced by the $r$-process; the dashed line shows that fraction for the solar $s$-process. process inventory of Ba for the star's $[\mathrm{Fe} / \mathrm{H}]$ value) achieved by the most extreme of the $s$-process-dominated stars. This large $\mathrm{Ba}$ enhancement is achieved at $[\mathrm{Fe} / \mathrm{H}] \sim-2.5$ dex. Comparing this, and the $\mathrm{Pb}$ enhancement of HE 21481247, with Figure 12 of Busso, Gallino, \& Wasserburg (1999) suggests that some adjustments are required in the model of galactic nucleosynthesis from AGB stars. Furthermore, these excesses in HE 2148-1247 must be confined mostly to the stellar surface. There cannot have been much sedimentation or mixing of the additional material into the interior of HE 2148-1247; otherwise, the amount of additional neutron capture material required would become prohibitively large.

Figure 13 suggests that the efficiency for the complex chain of events proposed to explain highly s-processenhanced very metal-poor stars, which includes nucleosynthesis in AGB stars, dredge-up to the surface of the AGB star, and mass transfer across a binary, can be surprisingly efficient; we have effectively taken a very low metallicity star and given it a substantial fraction of the solar sprocess abundances. This is a much more effective way of enhancing the abundances for a particular star in a binary system with a suitable mass ratio than is the production and distribution into the ISM of a single SN II. We speculate that all of the very metal-poor stars with more than $10 \%$ of the solar inventory of neutron capture elements must be (or have been) binaries. At least a few of the known extremely $s$-process-enhanced stars have been found to be binaries, in particular the two from the HES (HE 2148-1247, HE 0024-2523; Lucatello et al. 2003), LP 625-44 (Aoki et al. 2000, 2002b), as well as CS 29526-110 (Aoki et al. 2002a).

It appears that the relative amount of lead produced in such $s$-process events is not constant. While in some cases, including that of HE 2148-1247 and HE 0024-2523 (Lucatello et al. 2003), it is as large as was predicted by Gallino et al. (1998) and Busso et al. (1999), in other cases the $\mathrm{Pb}$ enhancement is more modest. As is widely recognized (see, e.g., Busso et al. 1999), the degree of $\mathrm{Pb}$ enhancement depends on the neutron to Fe seed ratio. Figure 15 displays the $[\mathrm{Pb} / \mathrm{Ba}]$ ratio as a function of the $s$-process enhancement (parameterized in this case by $[\mathrm{Ba} / \mathrm{Fe}]$ ) for the same set of stars shown in Figure 14, all stars with $[\mathrm{Fe} / \mathrm{H}] \leq-2.0$ dex. As noted by others, there is a considerable enhancement of $\mathrm{Pb}$ but well below the values to be expected if the neutron exposure were the same at low $[\mathrm{Fe} / \mathrm{H}]$ as for the solar mainstream $s$-process. It follows that the neutron source strength cannot be the same as calculated for the main "solar" $s$ process. Otherwise the conversion of $\mathrm{Fe}$ to $\mathrm{Pb}$ would be almost complete at $[\mathrm{Fe} / \mathrm{H}] \sim-2.4$. We infer that the neutron exposure roughly scales with $[\mathrm{Fe} / \mathrm{H}]$ but with some variation producing higher $\mathrm{Pb}$ but not greatly altering the $\mathrm{Ba}$ peak.

The origin of the apparent $r$-process enhancement in HE $2148-1247$ is, however, not known. Such a large $r$-process enhancement is very rare. The enhancement of Eu found among the pure $r$-process-enhanced stars is much lower (see Fig. 13). In previous studies it was argued that the high enrichments of $r$-process elements in a low-metallicity star must be the result of surface contamination by a binary companion subsequent to the formation of the binary and do not represent the composition of the interstellar medium (ISM) from which the star was formed (Qian \& Wasserburg 2001). Hence, one possible way to produce both $s$ - and 


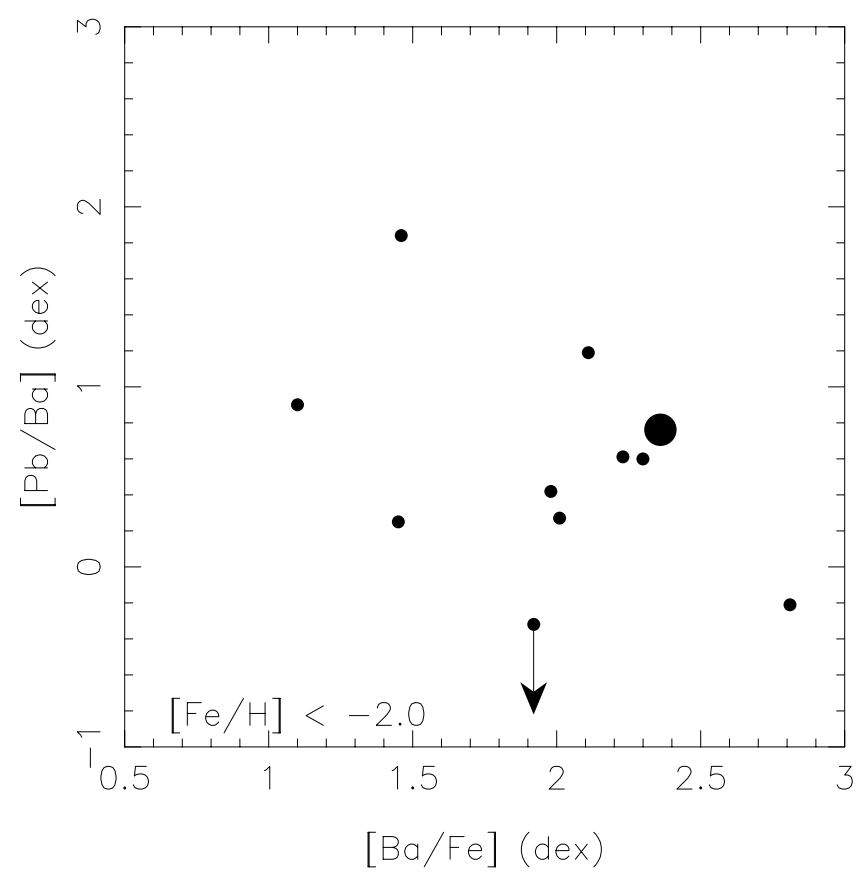

Fig. 15.-Enhancement of lead as compared to $\mathrm{Ba}$ is plotted as a function of the $s$-process enhancement (characterized by $[\mathrm{Ba} / \mathrm{Fe}]$ ) for very metal-poor $([\mathrm{Fe} / \mathrm{H}] \leq-2.0 \mathrm{dex}) s$-process-dominated stars. The same sample shown in Fig. 14, for which $\mathrm{Pb}$ abundances or upper limits could be located in the literature, is used. HE $2148-1247$ is indicated by the large filled circle.

$r$-enhancement is to consider that HE 2148-1247 was the least massive member of a triple system. It could then have obtained its $r$-process material from a massive star in this system that became a SN II. Then, assuming the binary did not become unbound by the SN explosion nor the low-mass star ablated away, the intermediate mass component of this system, as it passed through the AGB phase, could have produced the $s$-process material and the excess of $\mathrm{C}$ and $\mathrm{N}$, and transferred this material across the binary to $\mathrm{HE}$ 2148-1247. A triple star system with a history as described above appears to be an implausible scenario.

Another possibility is that our understanding of the $s$ process yields in AGB stars is flawed, and that all of the Eu as well as the Ba observed in HE 2148-1247 was produced through the $s$-process. The range of $\mathrm{Ba} / \mathrm{Eu}$ predicted from AGB nucleosynthesis in different calculations is fairly broad. Arlandini et al. (1999) predict $\mathrm{Ba} / \mathrm{Eu} 640$, which is slightly smaller than that of Käppeler et al. (1989) as quoted in Burris et al. (2000) for the solar $s$-process inventory, but this is still far larger than the ratio observed in $\mathrm{HE}$ 2148-1247. However, the predicted $\mathrm{Ba} / \mathrm{Eu}$ ratio for the most metal-poor case (and only that particular case) of AGB nucleosynthesis by Goriely \& Mowlavi (2000) of 105 is much closer to that of HE 2148-1247 than those of most other groups. In either case, the absolute abundances of the heavy elements predicted after dredge-up at the surface of a very metal-poor AGB star are insufficient to explain the observations. Furthermore, as is shown in Figure 13, very metal-poor stars with $[\mathrm{Fe} / \mathrm{H}]-2.2$ to -2.6 with enhanced abundances of the heavy neutron capture elements that appear dominated by the $s$-process show $\mathrm{Ba} / \mathrm{Eu}$ ratios of $300-800$, considerably larger than that of HE 2148-1247. Hence, this hypothesis too is rejected.
The theoretical calculations of $s$-process production via AGB nucleosynthesis, followed by dredge-up to the surface of the AGB star, then by mass transfer across a binary system, are very complex. As reviewed by Busso et al. (1999), models of nucleosynthesis in AGB stars have advanced greatly over the past few years, particularly at near solar metallicity, although the problem of galactic enrichment over a wide range of metallicity is not adequately addressed. However, it is clear that changes in AGB models to fit such an extreme case as HE 2148-1247 seem to be required.

\subsection{Accretion-induced Collapse, Two Stars Instead of Three}

Accretion-induced collapse (AIC) of a white dwarf in a binary is an interesting possibility to produce the enhancements seen in HE 2148-1247. This produces a bare neutron star, which also might satisfy the requirement for the source of the heavy $r$-elements. The AIC mechanisms were discussed earlier (e.g., Nomoto \& Kondo 1991), and the possibility of AIC events being the source of $r$-elements was pointed out by Woosley \& Baron (1992). The case for AIC events being a source of the heavy $r$-elements will be presented in a companion paper, Qian \& Wasserburg (2003). The data on HE 2148-1247 were critical in this inference. The overall model is that a binary system is initially formed with one star being of mass $M \sim 3-12 M_{\odot}$ and the other being of $M \lesssim 1 M_{\odot}$. The more massive star evolves through the AGB phase producing $s$-elements, some of which are transferred to its lower-mass companion. Depending on the ratio of neutrons to the $\mathrm{Fe}$ seeds in the AGB star, the $s$ process may produce an extremely high $\mathrm{Pb} / \mathrm{Ba}$ ratio or a pattern similar to the solar main $s$-pattern. The more massive star eventually loses its envelope and becomes a white dwarf, which upon accreting material from the companion, subsequently collapses into a neutron star. The surface layers of the neutron star are heated by an enormous flux of neutrinos. This drives a wind, in which the heavy $r$-elements are produced. Some of the heavy $r$-elements are again transferred to the companion. We consider this scenario to be a self-consistent explanation for the data on HE 2148-1247 and for the observations of other low-metallicity stars with high enrichments ranging from predominantly $r$-process to predominantly $s$-process in origin. Furthermore, we note that the binary might be disrupted during the AIC event. This would explain why a number of low-metallicity stars with high $s$-process enrichments appear to be single stars (Preston \& Sneden 2001).

\section{COMMENTS ON COSMOCHRONOLOGY}

$\mathrm{Th} / \mathrm{Eu}$ cosmochronology depends critically on the assumption that the initial production ratio of these two elements is constant and is that given by the $r$-process. Th/Eu for HE 2148-1247 is consistent with the expected (old) age for very metal-poor stars to within the (large) uncertainties. The Th/Eu values for the bright stars studied by Johnson \& Bolte (2001) and for BD $+17^{\circ} 3248$ (Cowan et al. 2002) are consistent as well.

However, CS 22892-052 has an apparent $\mathrm{Th} / \mathrm{Eu}$ age of 16 Gyr, while CS 31082-001 has a very young Th/Eu age (Hill et al. 2002). Among the possible causes of this are nonuniformities in the $r$-process yields (see, e.g., Otsuki, Matthews, \& Kajino 2002) or an underestimate in the errors 
in the abundance determinations for the elements Th and/ or Eu in these two stars. ${ }^{16}$ The first of the above difficulties, as well as the impact of potential $s$-process contributions to the Eu abundance such as occurs in HE 2148-1247, might be decreased considerably by moving from a chronology based on $\mathrm{Th} / \mathrm{Eu}$ to one based on $\mathrm{Th} / \mathrm{U}$, but detection of $\mathrm{U}$ is quite difficult.

Our analysis of HE 2148-1247 provides good evidence for mixing of $r$ - and $s$-process enhancements, which in turn leads to variation of the relative abundances of the heavy neutron capture elements, in particular $\mathrm{Ba} / \mathrm{Eu}$ and hence $\mathrm{Th} / \mathrm{Eu}$, in very metal-poor stars. We are still far from an understanding of the details of the nucleosynthesis sites and production mechanisms for the heavy elements in very metal-poor stars; hence, the use of $\mathrm{Eu} / \mathrm{Th}$ as a chronometer seems fraught with difficulties.

The entire Keck/HIRES user community owes a huge debt to Jerry Nelson, Gerry Smith, Steve Vogt, and many Gyr.

${ }^{16}$ An uncertainty of 0.10 dex corresponds to an age uncertainty of $\sim 4$ other people who have worked to make the Keck Telescope and HIRES a reality and to operate and maintain the Keck Observatory. We are grateful to the W. M. Keck Foundation for the vision to fund the construction of the W. M. Keck Observatory. This publication makes use of data products from the Two Micron All Sky Survey, which is a joint project of the University of Massachusetts and the Infrared Processing and Analysis Center/California Institute of Technology, funded by the National Aeronautics and Space Administration and the National Science Foundation. We thank C. Conselice and I. Ivans for acquiring the direct images used for photometry and A. McWilliam for providing a code to calculate hfs. J. G. C. is grateful for partial support from NSF grants AST-9819614 and AST0205951. N. C. acknowledges financial support through a Marie Curie Fellowship of the European Community program Improving Human Research Potential and the Socio-Economic Knowledge under contract number HPMFCT-2002-01437, and from Deutsche Forschungsgemeinschaft under grant Re 353/44-1. Y. Z. Q. is supported by DOE grants DE-FG02-87ER40328 and DE-FG0200ER41149, and G. J. W. by NASA grant NAG5-11725.
Allende-Prieto, C., Lambert, D. L., \& Asplund, M. 2001, ApJ, 556, L63 2002, ApJ, 573, L137

Anders, E., \& Grevesse, N. 1989, Geochim. Cosmochim. Acta, 53, 197

Aoki, W., Norris, J. E., Ryan, S. G., Beers, T. C., \& Ando, H. 2000, ApJ, 536, L97

Aoki, W., Ryan, S. G., Norris, J. E., Beers, T. C., Ando, H., \& Tsangarides, S. $2002 \mathrm{a}$, ApJ, 580, 1149

Aoki, W., et al. 2001, ApJ, 561, 346 $2002 \mathrm{~b}$, PASJ, 54,427

Arlandini, C., Käppeler, F., Wisshak, K., Gallino, R., Lugaro, M., Busso, M., \& Straniero, O. 1999, ApJ, 525, 886

Arnesen, A., et al. 1982, A\&A, 106, 327

Asplund, M., Nordlund, A., Trampedach, R., Prieto, C. A., \& Stein, R. F. 2000, A\&A, 359, 729

Barklem, P. S., Piskunov, N., \& O’Mara, B. J. 2000, A\&AS, 142, 467

Baumüller, D., \& Gehren, T. 1996, A\&A, 307, 961

. 1997, A\&A, 325, 1088

Beers, T. C., Preston, G. W., \& Shectman, S. 1985, AJ, 90, 2089 1992 AJ, 103, 1987

Behr, B. B., Cohen, J. G., \& McCarthy, J. K. 2000, ApJ, 531, L37

Biémont, E., Garnir, H. P., Palmeri, P. Li, Z. S., \& Svanberg, S. 2000, MNRAS, 312, 116

Booth, A. J., Shallis, M. J., \& Wells, M. 1983, MNRAS, 205, 191

Burris, D. L., Pilachowski, C. A., Armandroff, T. E., Sneden, C., Cowan, J. J., \& Roe, H. 2000, ApJ, 544, 302

Busso, M., Gallino, R., Lambert, D. L., Travaglio, C., \& Smith, V. V. 2001, ApJ, 557, 802

Busso, M., Gallino, R., \& Wasserburg, G. J. 1999, ARA\&A, 37, 239

Carretta, E., Gratton, R. G., Cohen, J. G., Beers, T. C., \& Christlieb, N. 2002, AJ, 124, 481

Cayrel, R., et al. 2001, Nature, 409, 691

Childs, W. J., Poulsen, O., Goodman, L. S., \& Crosswhite, H. 1979, Phys. Rev. A, 19, 168

Christlieb, N., Wisotzki, L., \& Grasshoff, G. 2002, A\&A, 391, 397

Christlieb, N., Wisotzki, L., Reimers, D., Homeier, D., Koester, D., \& Heber, U. 2001, A\&A, 366, 898

Cohen, J. G., Christlieb, N., Beers, T. C., Gratton, R. G., \& Carretta, E. 2002, AJ, 124, 470

Cowan, J. J., et al. 2002, ApJ, 572, 861

Cowley, C. R., Ryabchikova, T., Kupka, F., Bord, D. J., Mathys, G., \& Bidelman, W. P. 2000, MNRAS, 317, 299

Fuhr, J. R., Martin, G. A., \& Wiese, W. L. 1988, J. Phys. Chem. Ref. Data, 17,4

Fujimoto, M. Y., Ikeda, Y., \& Iben Jr., I. 2000, ApJ, 529, L25

Gallino, R., Arlandini, C., Buzzo, M., Lugaro, M., Travaglio, C., Straniero, O., Chieffi, A., \& Limongi, M. 1998, ApJ, 497, 388

Gehren, T., Butler, K., Mashonkina, L., Reetz, J., \& Shi, J. 2001, A\&A, 366, 981

Gehren, T., Korn, A. J., \& Shi, J. 2001, A\&A, 380, 645

Goriely, S., \& Mowlavi, N. 2000, A\&A, 362, 599

Goriely, S., \& Siess, L. 2001, A\&A, 378, L25

Gratton, R. G., Carretta, E., Eriksson, K., \& Gustafsson, B. 1999, A\&A, 350,955

\section{EFERENCES}

Grevesse, N., \& Sauval, A. J. 1998, Space Sci. Rev., 85, 161

Gustafsson, B., Bell, R. A., Eriksson, K., \& Nordlund, A. 1975, A\&A, 42, 407

Han, Z., Eggleton, P. P., Podsiadlowski, P., \& Tout, C. A. 1995, MNRAS, 277,1443

Hill, V., et al. 2000, A\&A, 353, 557 2002, A\&A, 387, 560

Holweger, H. 2001, in AIP Conf. Proc. 598, Solar and Galactic Composition, ed. R. F. Wimmer-Schweingruber (New York: AIP), 23

Holweger, H., Bard, A., Kock, A., \& Kock, M. 1991, A\&A, 249, 545

Houdashelt, M. L., Bell, R. A., \& Sweigart, A. V. 2000, AJ, 119, 1448

Johnson, J. A., \& Bolte, M. 2001, ApJ, 554, 888

Jorgensen, U. G. 1994, A\&A, 284, 179

Jorgensen, U. G., Larsson, M., Iwamae, A., \& Yu, B. 1996, A\&A, 315, 204

Jorissen, A., \& Boffin, H. M. J. 1992, in Binaries as Tracers of Star Formation, ed. A. Duquennoy \& M. Mayor (Cambridge: Cambridge Univ. Press), 110

Käppeler, F., Beer, H., \& Wisshak, K. 1989, Rep. Prog. Phys., 52, 945

Korn, A. J., \& Gehren, T. 2003, in IAU Symp. 210, Modeling of Stellar Atmospheres, ed. N. Piskunov, W. W. Wiess, \& D. F. Gray (San Francisco: ASP), in press

Kurucz, R. L. 1993, Kurucz CD-ROM 13, ATLAS 9 Stellar Atmosphere Programs and $2 \mathrm{~km} / \mathrm{s}$ Grid (Cambridge: $\mathrm{SAO}$ )

. 1994, Kurucz CD-Rom 15, Diatomic Molecular Data (Cambridge: SAO)

. 1995, Kurucz CD-Rom 23, Atomic Line List (Cambridge: SAO)

Lawler, J. E., Bonvallet, G., \& Sneden, C. 2001a, ApJ, 556, 452

Lawler, J. E., \& Dakin, T. 1989, J. Opt. Soc. Am. B, 6, 1457

Lawler, J. E., Whaling, W., \& Grevesse, N. 1990, Nature, 346, 635

Lawler, J. E., Wickliffe, M. E., Den Hartog, E. A., \& Sneden, C. 2001b, ApJ, 563, 1075

Lucatello, S., Gratton, R., Cohen, J. G., Beers, T. C., Christlieb, N., Carretta, E., \& Ramírez, S. 2003, AJ, 125, 875

Magain, P. 1984, A\&A, 134, 189

Mansour, N. B., Dinneen, T., Young, L., \& Cheng, K. T. 1989, Phys. Rev. A, 39, 5762

Martin, G. A., Fuhr, J. R., \& Wiese, W. L. 1988, J. Phys. Chem. Ref. Data, 17,3

Mashonkina, L., Gehren, T., \& Bikmaev, I. 1999, A\&A, 343, 519

McCarthy, J. K. 1988, Ph.D. thesis, Caltech

McClure, R. B. 1983, ApJ, 268, 264

McWilliam, A. 1998, AJ, 115, 1640

McWilliam, A., Preston, G. W., Sneden, C., \& Shectman, S. 1995, AJ, 109, 2736

Morrell, O., Källander, D. \& Butcher, H. R. 1992, A\&A, 259, 543

Nomoto, K., \& Kondo, Y. 1991, ApJ, 367, L19

Norris, J. E., Ryan, S. G., \& Beers, T. C. $1997 \mathrm{a}$, ApJ, 488, 350

Otsuki, K., Mathews, G. J., \& Kajino, T. 2002, ApJ, submitted (astro-ph/ 0207596)

Pickering, J. C. 1996, ApJS, 107, 811

Pickering, J. C., \& Thorne, A. P. 1996, ApJS, 107, 761

Preston, G. W., \& Sneden, C. 2001, AJ, 122, 1545 
Prochascka, J. X., Naumov, S. O., Carney, B. W., McWilliam, A., \& Wolfe, A. M. 2000, AJ, 120, 2513

Qian, Y.-Z., \& Wasserburg, G. J. 2001, ApJ, 552, L55 2002, ApJ, 567, 515

.2003, ApJ, submitted

Ramírez, S. V., \& Cohen, J. G. 2003, AJ, 125, 224

Ramírez, S. V., Cohen, J. G., Buss, J., \& Briley, M. M. 2001, AJ, 122, 1429

Schlegel, D. J., Finkbeiner, D. P., \& Davis, M. 1998, ApJ, 500, 525

Shortridge, K. 1993, in ASP Conf. Ser. 52, Astronomical Data Analysis Software and Systems II, ed. R. J. Hannisch, R. J. V. Brissenden, \& J. Barnes (San Francisco: ASP), 219

Siess, L., Livio, M., \& Lattanzio, J. 2002, ApJ, 570, 329

Simons, J. W., Palmer, B. A., Hof, D. E., \& Oldenborg, R. C. 1989, J. Opt. Soc. Am. B, 6, 1097

Skrutskie, M. F., et al. 1997, in The Impact of Large-Scale Near-IR Sky Surveys, ed. F. Garzon et al. (Dordrecht: Kluwer), 187

Sneden, C. 1973, Ph.D. thesis, Univ. Texas

Sneden, C., Cowan, J. J., Ivans, I., Fuller, G. M., Burles, S., Beers, T. C., \& Lawler, J. E. 2000, ApJ, 533, L139

Sneden, C., McWilliam, A., Preston, G. W., Cowan, J. J., Burris, D. L., \& Armosky, B. J. 1996, ApJ, 467, 819

Sneden, C., Preston, G. W., McWilliam, A., \& Searle, L. 1994, ApJ, 431, L27
Thévenin, F., \& Idiart, T. P. 1999, ApJ, 521, 753

Unsöld, A. 1955, Physik der Sternatmospharen (2d ed.; Berlin: Springer)

Van Eck, S., Goriely, S., Jorissen, A., \& Plez, B. 2001, Nature, 412, 793

Villemoes, P., van Leeuwen, R., Heijkenskjöld, F., Kastberg, A., Larsson, M. O., \& Kotochigova, S. A. 1992, Phys. Rev. A, 45, 6241

Vogt, S. E., et al. 1994, Proc. SPIE, 2198, 362

Wallace, L., Hinkle, K., \& Livingston, W. C. 1998, An Atlas of the Spectrum of the Solar Photosphere from 13,500 to $28,000 \mathrm{~cm}^{-1}$ (35707405 A) (NSO Tech. Rep. 98-001; Tucson: National Solar Obs.)

Westin, J., Sneden, C., Gustafsson, B., \& Cowan, J. J. 2000, ApJ, 530, 783

Wiese, W. L., Fuhr, J. R., \& Deters, T. M. 1996, J. Phys. Chem. Ref. Data, 7,1

Wiese, W. L., Smith, M. W., \& Miles, B. M. 1969, Atomic Transition Probabilities, Vol. 2, Sodium through Calcium (Washington, DC: GPO)

Wisotzki, L., Christlieb, N., Bade, N.,Beckmann, V., Köhler, T., Vanelle, C., \& Reimers, D. 2000, A\&A, 358, 77

Woosley, S. E., \& Baron, E. 1992, ApJ, 391, 228

Yi, S., Demarque, P., Kim, Y. C., Lee, Y. W., Ree, C. H., Lejeune, T., \& Barnes, S. 2001, ApJS, 136, 417

Zacs, L., Nissen, P. E., \& Schuster, W. J. 1998, A\&A, 337, 216

Zhao, G., Butler, K., \& Gehren, T. 1998, A\&A, 333, 219

Zhao, G., \& Gehren, T. 2000, A\&A, 362, 1077 